\title{
SIMPLE LINEAR TIME ALGORITHMS FOR PIERCING PAIRWISE INTERSECTING DISKS
}

YUNKAI WANG

A thesis submitted to the Faculty of Graduate and Post Doctoral Affairs

in partial fulfillment of the requirements for the degree of

\section{Master of Computer Science}

\section{Carleton University}

Ottawa, Ontario, Canada 
In this thesis, we study the problem of piercing pairwise intersecting disks in the plane. A set $\mathcal{D}$ of disks is said to be pierced by a point set $P$ if each disk in $\mathcal{D}$ contains a point of $P$. Any set of pairwise intersecting unit disks can be pierced by 3 points (H. Hadwiger and $\mathrm{H}$. Debrunner, Ausgewählte Einzelprobleme der kombinatorischen Geometrie in der Ebene, Enseignement Math, 1955), and any set of pairwise intersecting disks of arbitrary radius can be pierced by 4 points (L. Danzer, Zur Lösung des Gallaischen Problems über Kreisscheiben in der Euklidischen Ebene, Studia Scientiarum Mathematicarum Hungarica, 1986). However, these proofs do not lend themselves to efficient algorithms for computing a piercing set. Recently, linear-time algorithms for finding the piercing points have been proposed. These algorithms compute the piercing points based on either the smallest destroyer of $\mathcal{D}$ or the smallest disk that intersects every disk in $\mathcal{D}$. The computation of the smallest destroyer of $\mathcal{D}$ or the smallest disk that intersects every disk in $\mathcal{D}$ can be formulated as an LP-type problem. We present a simple deterministic linear-time algorithm for finding 3 piercing points for pairwise intersecting unit disks. We also present a simple deterministic linear-time algorithm for finding 5 points that pierce any set of pairwise intersecting disks of arbitrary radius. Our algorithms use simple geometric transformation and avoid LP-type machinery. In this thesis, we also present a set of 9 pairwise intersecting unit disks that cannot be pierced by 2 points, which shows that 3 points are sometimes necessary and always sufficient to pierce pairwise intersecting unit disks. 


\section{ACKNOWLEDGMENTS}

Firstly, I would like to express my very great appreciation to my cosupervisors Prosenjit Bose and Ahmad Biniaz for their guidance, support, helpful discussions, and many reviews of and improvements to the many drafts. Thanks to the examination committee Jean-Lou De Carufel and Pat Morin for their helpful comments.

Thanks to my family and friends for their love and support.

Thanks to André Miede for the $\mathrm{LT}_{\mathrm{E} X} \mathrm{X}$ thesis template.

Finally, thanks to you for reading this. Stay home and stay safe.= ) 
CONTENTS

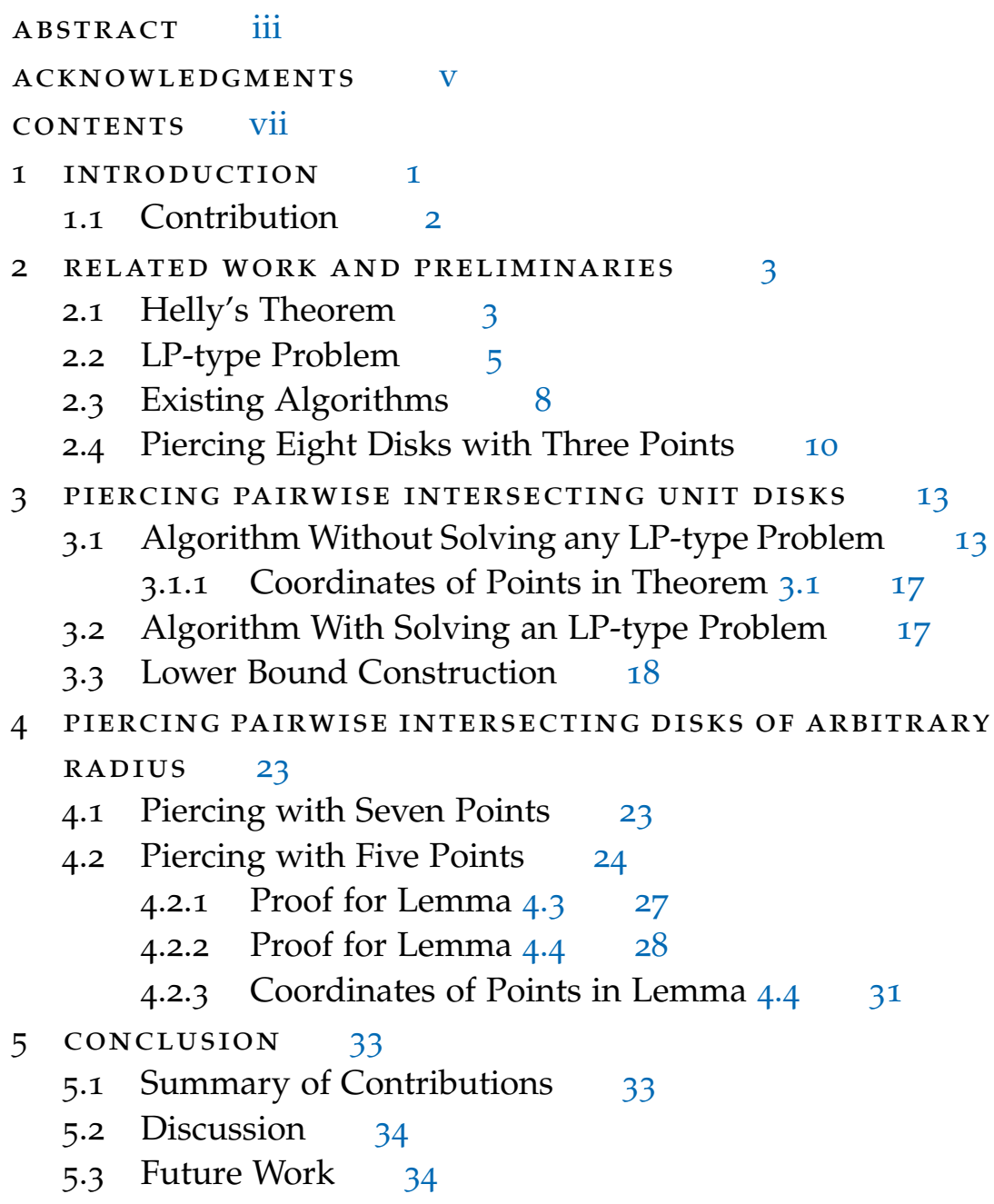


In this thesis, we focus on piercing pairwise intersecting disks in the plane.

Let $\mathcal{D}$ be a set of pairwise intersecting disks in the plane. Our goal is to pierce $\mathcal{D}$ using a set of points of minimum cardinality. If every set of 3 disks in $\mathcal{D}$ has a nonempty intersection, then the whole set has a nonempty intersection, and $\mathcal{D}$ can be pierced by 1 point [16]. This result is known as Helly's Theorem. We will give a formal definition of Helly's Theorem in Section 2.I. A finite collection $\mathcal{D}$ of disks is Helly if their common intersection is nonempty; otherwise, we say that $\mathcal{D}$ is non-Helly.

When all pairs of disks in $\mathcal{D}$ intersect, but $\mathcal{D}$ contains groups of 3 disks that have no common intersection, then $\mathcal{D}$ is non-Helly and thus finding a set of points that pierces $\mathcal{D}$ becomes more difficult. Hadwiger and Debrunner first showed that if all the disks in $\mathcal{D}$ have the same radius, then there always exists a set of 3 points in the plane that pierce $\mathcal{D}$ [13]. Their construction is based on a regular hexagon that covers the centers of all the disks in $\mathcal{D}$.

When the disks in $\mathcal{D}$ have different radii, 3 points are sometimes insufficient $[12,8,15]$. Danzer and Stachó independently proved that 4 points are always sufficient for piercing any finite set of pairwise intersecting disks in the plane. Danzer published his proof in 1986 [8]. His proof uses a compactness argument and part of the proof is based on an undetailed verification by the computer. Stachó's proof [23] is based on three disks in $\mathcal{D}$ that are non-Helly and their inscribed circle is the largest. The inscribed circle of three disks is the smallest circle that is tangent to all three disks. It is not known whether we can find such three disks in $\mathcal{D}$ in linear time.

In 2018, Har-Peled et al. [15] gave a new proof for the existence of 5 piercing points for any finite set of pairwise intersecting disks of arbitrary radius. Their proof is constructive and it computes the piercing points using the smallest destroyer of $\mathcal{D}$. Let $\mathcal{D}=\left\{\mathrm{D}_{1}, \mathrm{D}_{2}, \ldots, \mathrm{D}_{\mathrm{n}}\right\}$ in the order of increasing radius. $D_{i}$ is the smallest destroyer of $\mathcal{D}$ when $D_{i}$ is the smallest disk in $\mathcal{D}$ such that $\left\{D_{1}, D_{2}, \ldots, D_{i-1}\right\}$ is a Helly set. The problem of finding the smallest destroyer is LP-type. We will introduce LP-type problems in Section 2.2.

Meanwhile, Carmi et al. [3] presented a linear-time algorithm for finding 4 points that pierce a set of pairwise intersecting disks of arbitrary radius. Their algorithm computes the piercing points based on the smallest disk intersecting every disk in $\mathcal{D}$. Finding such disk is also an LP-type problem. Their algorithm considers 8 different cases. 
In each of these cases, they compute 4 points that pierce $\mathcal{D}$ based on the settings of the disks in $\mathcal{D}$.

As for the lower bound on this problem, Grünbaum [12] provided a set of 21 pairwise intersecting disks that cannot be pierced by 3 points. Later in time, Danzer [8] reduced the number of disks to 10. However, Danzer's construction is difficult to verify since the positions of the disks cannot be visualized easily. Har-Peled et al. [15] gave a simpler lower bound construction of 13 disks whose positions can be easily visualized.

In the rest of the thesis, in Section 1.I, we will talk about the contribution of this thesis. In Chapter 2, we will discuss all the related work and preliminaries. In Chapter 3 , we describe our linear-time algorithms for piercing pairwise intersecting unit disks and our lower bound construction. Chapter 4 will contain a detailed explanation of our proposed linear-time algorithm for piercing pairwise intersecting disks of arbitrary radius. Finally, Chapter 5 will briefly summarize all the results and discuss possible directions for future work.

\section{I CONTRIBUTION}

In this thesis, we will present two deterministic linear-time algorithms for finding 3 points that pierce any set of pairwise intersecting unit disks. Both of our algorithms do not build the set of points based on the regular hexagon covering the centers of all the disks. The first algorithm uses simple geometric transformation and computes the piercing points using only the positions of the disks in the set. The second algorithm computes the piercing points using the smallest disk that intersects every disk in the set. The analysis for both algorithms is simple and both algorithms run in linear time.

For the lower bound on pairwise intersecting unit disks, we present a set of 9 unit disks. These 9 disks are pairwise intersecting and they cannot be pierced by 2 points. This completes the proof that 3 is the optimal number of points for piercing any set of pairwise intersecting unit disks.

We also present a deterministic linear-time algorithm for finding 5 points that pierce any finite set of pairwise intersecting arbitrary disks. Our algorithm avoids heavy machinery by not involving any LP-type problem. Our algorithm starts by shrinking the smallest disk until it becomes tangent to a second disk in the set, then it computes two candidate sets of 5 points using only the information of these two disks. The computation of the coordinates of these points takes constant time. One of the two candidate sets guarantees to pierce the set of disks. 
In this chapter, we discuss all the related works and preliminaries.

Researchers have been studying the piercing problem since 1913. Let $X$ be a set of convex subsets in $\mathbb{R}^{\mathrm{d}}$. In 1913, Eduard Helly [9] proved that if every $d+1$ convex subsets of $X$ in $\mathbb{R}^{d}$ has a nonempty intersection, then the whole set $X$ has a nonempty intersection. Helly never published the result until 1923 [16], by which time alternate proofs had been given by Radon [20] and König [17]. We will give a formal definition of Helly's Theorem and prove it using induction in Section 2.1.

Later in time, Helly's Theorem was significantly generalized and extended. In 1992, Alon and Kleitman [1] proved the generalized $(p, q)$-theorem. Let $\mathcal{F}$ be a set of convex subsets in $\mathbb{R}^{d}$. $(p, q)$-theorem states that if among any $p$ members of the convex sets $\mathcal{F}$ in $\mathbb{R}^{\mathrm{d}}$, some $q$ has a nonempty intersection where $p \geqslant q \geqslant d+1$, then there exists a set of at most $c$ points where $c<\infty$ that pierces $\mathcal{F}$. Helly's Theorem is one special case of the generalized $(p, q)$-theorem where $p=q=d+1$. For interested reader, please refer to the reference material [1].

We will focus on piercing pairwise intersecting disks. Let $\mathcal{D}$ be a set of pairwise intersecting disks in the plane. If all the disks in $\mathcal{D}$ have the same radius, then up to scaling we assume they all have unit radius. If the disks in $\mathcal{D}$ have different radii, then we sometimes refer to $\mathcal{D}$ as a set of arbitrary disks. If $\mathcal{D}$ is Helly, then the problem of finding a point that lies in $\cap \mathcal{D}$ is LP-type [18]. An LP-type problem is an abstract generalization of a low-dimensional linear program [6, 19]. We will describe in detail what is an LP-type problem in Section 2.2.

The linear-time algorithms proposed by Har-Peled et al. [15] and Carmi et al. [3] for piercing pairwise intersecting arbitrary disks both use the LP-type problem as a subroutine. In section 2.3, we describe in more details how their algorithms work and why solving an LP-type problem is mandatory in their algorithms.

This chapter ends with Section 2.4 in which we show that every set of 8 pairwise intersecting disks of arbitrary radius can be pierced by 3 points.

\subsection{HELLY'S THEOREM}

In this section, we talk about Helly's Theorem. We will prove Helly's Theorem using Radon's Theorem as in the proof by Radon [20]. Let 
$\operatorname{conv}(\mathcal{P})$ be the convex hull of $\mathcal{P}$ where $\mathcal{P}$ is a set of points in $\mathbb{R}^{\mathrm{d}}$ and let $\overrightarrow{0}$ represents the 0 -vector in $\mathbb{R}^{\mathrm{d}}$.

THEOREM 2.1 (Radon's Theorem). Given any set $\mathcal{P}$ of $\mathrm{d}+2$ points in $\mathbb{R}^{\mathrm{d}}$, one can partition $\mathcal{P}$ into two sets $\mathcal{P}_{1}$ and $\mathcal{P}_{2}$, such that $\operatorname{conv}\left(\mathcal{P}_{1}\right) \cap$ $\operatorname{conv}\left(\mathcal{P}_{2}\right) \neq \emptyset$.

Proof. Let $\mathcal{P}=\left\{p_{1}, p_{2}, \ldots, p_{d+2}\right\}$ be the set of points. We can find real numbers $a_{1}, a_{2}, \ldots, a_{d+2}$, not all of them are zero, such that

$$
\sum_{i} a_{i} p_{i}=\overrightarrow{0}, \sum_{i} a_{i}=0 .
$$

To show this, consider the $d+1$ points $p_{2}-p_{1}, p_{3}-p_{1}, \ldots, p_{d+2}-p_{1}$, they are linearly dependent, so there must exist $b_{3}, b_{4}, \ldots, b_{d+2}$ such that

$$
p_{2}-p_{1}=\sum_{i=3}^{d+2} b_{i}\left(p_{i}-p_{1}\right) .
$$

By rearranging terms in the equation, we get

$$
\left(b_{3}+\cdots+b_{d+2}-1\right) p_{1}+p_{2}+\left(-b_{3}\right) p_{i}+\cdots+\left(-b_{d+2}\right) p_{d+2}=0
$$

Set $a_{1}=b_{3}+\cdots+b_{d+2}-1, a_{2}=1, a_{i}=-b_{i}$ where $i \geqslant 3$.

Let $A_{1}=\left\{i \mid a_{i}>0\right\}, A_{2}=\left\{i \mid a_{i} \leqslant 0\right\}, \mathcal{P}_{1}=\left\{p_{i}: i \in A_{1}\right\}$ and $\mathcal{P}_{2}=\left\{p_{i}: i \in A_{2}\right\}$ where $i \in\{1, \ldots, d+2\}$. Both $A_{1}$ and $A_{2}$ are nonempty since $\sum_{i} a_{i}=0$. Set $S=\sum_{i \in A_{1}} a_{i}$. The point $x=\sum_{i \in A_{1}} \frac{a_{i} p_{i}}{S}$ lies in $\operatorname{conv}\left(\mathcal{P}_{1}\right) . \sum_{i \in \mathrm{P}_{1}} a_{i} p_{i}+\sum_{i \in P_{2}} a_{i} p_{i}=0$, we have that $x=$ $\sum_{i \in A_{2}} \frac{-a_{i} p_{i}}{S}$, so $x$ also lies in $\operatorname{con} v\left(\mathcal{P}_{2}\right)$. Therefore, $x \in \operatorname{conv}\left(\mathcal{P}_{1}\right) \cap$ $\operatorname{conv}\left(\mathcal{P}_{2}\right)$. Any points that lies in $\operatorname{conv}\left(\mathcal{P}_{1}\right) \cap \operatorname{conv}\left(\mathcal{P}_{2}\right)$ is called a Radon Point.

We now show how we can use Radon's Theorem to prove Helly's Theorem. We will again use the proof given by Radon [20]. The proof is by induction on $n$ : the number of convex subsets in $X$.

THEOREM 2.2 (Helly's Theorem). Let $X=\left\{X_{1}, X_{2}, \ldots, X_{n}\right\}$ be a set of convex subsets of $\mathbb{R}^{\mathrm{d}}$ where $\mathrm{n}>\mathrm{d}+1$. If the intersection of every $\mathrm{d}+1$ of these sets is nonempty, then the whole set $X$ has a nonempty intersection.

Proof. Base case: When $\mathrm{n}=\mathrm{d}+2$, we want to show that one point suffice to pierce $X$. Since every group of $d+1$ convex subset of $X$ has a nonempty intersection, for $j \in\{1, \ldots, n\}$, we can find a point $x_{j}$ that lies in the common intersection of $\mathcal{X} \backslash x_{j}$. Let $\mathcal{P}=\left\{x_{1}, x_{2}, \ldots, x_{n}\right\}$. Theorem 2.I states that any set of $d+2$ points in $\mathbb{R}^{d}$ can be partitioned into two sets whose convex hulls intersect. Therefore, we can partition $\mathcal{P}$ into $\mathcal{P}_{1}$ and $\mathcal{P}_{2}$ and find a Radon Point $p$ that falls in the intersection of the convex hulls of $\mathcal{P}_{1}$ and $\mathcal{P}_{2}$. We want to prove that $p$ pierces $X$. We first notice that the only element of $\mathcal{P}$ that may not be in $X_{j}$ is $x_{j}$. If $x_{j}$ is in $X_{j}$, then $p$ clearly lies in $X_{j}$. So we assume 
$x_{j} \notin X_{j}$ for some $j \in\{1, \ldots, n\}$. If $x_{j} \in \mathcal{P}_{1}$, then $x_{j} \notin \mathcal{P}_{2}$ and $X_{j} \supset \mathcal{P}_{2}$. Since $X_{j}$ is convex, the convex hull of $\mathcal{P}_{2}$ is contained in $X_{j}$, and thus the Radon Point lies in $X_{j}$. The argument is symmetric when $x_{j} \in \mathcal{P}_{2}$.

Inductive step: We first replace $X_{n-1}$ and $X_{n}$ with $X_{n-1} \cap X_{n}$ and $X_{n-1} \cap X_{n}$ is convex. Every $d+1$ convex subsets in the new set still has a nonempty intersection. Therefore we have a new collection $X^{\prime}$ of $n-1$ convex subsets. So, we can apply the inductive hypothesis which indicates that $\cap X^{\prime}$ is nonempty. Finally, if $\cap X^{\prime}$ is nonempty, then $\cap X$ must be nonempty since $\cap X^{\prime}=\cap X$.

To compute the piercing point, we can repeatedly replace two convex subsets in $X$ with their intersection until there are $d+2$ convex subsets left in the set. Then we compute the point set $\mathcal{P}$ as described in the proof of theorem 2.2. The time it takes to compute $\mathcal{P}$ depends on the representation of the convex subsets. The Radon Point can then be computed in polynomial time given $\mathcal{P}$ by using Gaussian elimination [11] or other efficient algorithms [7].

\subsection{LP-TYPE PROBLEM}

In this section, we will introduce the LP-type framework [22]:

DEFINITION 2.3 (The LP-type framework). An LP-type framework $(\mathcal{H}, w, \leqslant)$ is an abstract generalization of a low-dimensional linear program. It is made up of a finite set of constraints $\mathcal{H}$, a weight function $w: 2^{\mathcal{H}} \rightarrow \mathcal{W}$, and a total order $(\mathcal{W}, \leqslant)$ on the weights. The weight function $w$ assigns $a$ weight to each subset of constraints and it must satisfy these three axioms:

- Monotonicity: for any $\mathcal{H}^{\prime} \subseteq \mathcal{H}$ and $\mathrm{H} \in \mathcal{H}$, we have $w\left(\mathcal{H}^{\prime} \cup\{\mathrm{H}\}\right) \leqslant$ $w\left(\mathcal{H}^{\prime}\right)$.

- Finite Basis: there is a constant $\mathrm{d} \in \mathbb{N}$ such that for any $\mathcal{H}^{\prime} \subseteq \mathcal{H}$, there is a subset $\mathcal{B} \subseteq \mathcal{H}^{\prime}$ with $|\mathcal{B}| \leqslant \mathrm{d}$ and $w(\mathcal{B})=w\left(\mathcal{H}^{\prime}\right)$; and

- Locality: for any $\mathcal{B} \subseteq \mathcal{H}^{\prime} \subseteq \mathcal{H}$ with $w(\mathcal{B})=w\left(\mathcal{H}^{\prime}\right)$ and for any $\mathrm{H} \in \mathcal{H}$, we have that if $w(\mathcal{B} \cup\{\mathrm{H}\})=w(\mathcal{B})$, then also $w\left(\mathcal{H}^{\prime} \cup\{\mathrm{H}\}\right)=$ $w\left(\mathcal{H}^{\prime}\right)$.

A basis for a subset $\mathcal{H}^{\prime} \subseteq \mathcal{H}$ is an inclusion-minimal set $\mathcal{B} \in \mathcal{H}^{\prime}$ with $w(\mathcal{B})=w\left(\mathcal{H}^{\prime}\right)$. We know from the finite basis axiom that there are at most $d$ constraints in each basis. The goal of an LP-type problem is to determine $w(\mathcal{H})$ and find a basis $\mathcal{B}$ for $\mathcal{H}$.

Seidel's algorithm for low-dimensional linear programs [21] shows that LP-type problems can be solved in $\mathrm{O}(|\mathcal{H}|)$ expected time if we have a constant time violation test. Given a set $\mathcal{B} \subseteq \mathcal{H}$ and $H \in \mathcal{H}$, a violation test reports whether $H$ violates $\mathcal{B}$ and $H$ violates $\mathcal{B}$ if and only if $w(\mathcal{B} \cup\{\mathrm{H}\})<w(\mathcal{B})$. Chazelle and Matoušek [6] later showed that LP-type problems can be solved in $\mathrm{O}(|\mathcal{H}|)$ deterministic time if in addition to having a constant time violation test, the range 
space $\left(\mathcal{H},\left\{\right.\right.$ vio $\left(\mathcal{B} \mid \mathcal{B}\right.$ is a basis for some $\left.\left.\mathcal{H}^{\prime} \subseteq \mathcal{H}\right\}\right)$ has bounded VCdimension. Range space refers to a hypergraph and the VC-dimension of a range space represents the maximum cardinality of a shattered subset of the range space. The range space and the VC-dimension are outside the scope of this thesis, the interested reader should refer to the reference material [14]. For a basis $\mathcal{B}$, vio $(\mathcal{B}) \subset \mathcal{H}$ consists of all constraints that violate $\mathcal{B}$.

We can determine whether a given set of disks is Helly by formulating it as an LP-type problem. This result is derived from the theorem proved by Löffler and van Kreveld [18]:

LEMMA 2.4. (Theorem 6 in [18]). Given a set of $\mathrm{n}$ disks in the plane, the problem of finding the smallest disk that intersects every input disk can be solved in $\mathrm{O}(\mathrm{n})$ time.

The proof of lemma 2.4 uses the LP-type framework by Sharir and Welzl $[5,22]$. If the output disk has radius 0 , the given set of disks is Helly. Otherwise, the set is non-Helly. The algorithm by Carmi et al. [3] uses this result and computes the piercing points based on the smallest disk that intersects every disk in $\mathcal{D}$.

Chan [4] first observed that finding three disks in the set such that these three disks are non-Helly and one of these three disks is the smallest destroyer is an LP-type problem. However, he did not prove his claim. Har-Peled et al. [15] provided a proof for the claim in their paper. Before we come to their proof, we first refine some crucial definitions that are defined by Har-Peled et al. [15]:

DEFinition 2.5 (Helly). A finite set $\mathcal{D}$ of disks is Helly if the whole set has a nonempty intersection. Otherwise, we say that $\mathcal{D}$ is non-Helly. If $|\mathcal{D}|=3$ and $\mathcal{D}$ is Helly, then we denote $\mathcal{D}$ as Helly triple. Similarly, if there are 3 disks in $\mathcal{D}$ and $\mathcal{D}$ is non-Helly, then we denote $\mathcal{D}$ as non-Helly triple.

DEFINITION 2.6 (Destroyer). Let $\mathcal{D}$ be a set of pairwise intersecting arbitrary disks. A disk $\mathrm{D}$ is a destroyer of $\mathcal{D}$ if $\mathcal{D} \cup\{\mathrm{D}\}$ is non-Helly. If $\mathcal{D}$ is non-Helly, then every disk is a destroyer of $\mathcal{D}$.

DEFINITION 2.7 (Smallest Destroyer). Let $\mathcal{D}$ be a set of pairwise intersecting arbitrary disks. Let $\mathcal{D}_{<\mathrm{r}}$ be the set of all the disks in $\mathcal{D}$ whose radius are smaller than $r$. A disk $\mathrm{D} \in \mathcal{D}$ with radius $r$ is called a smallest destroyer of $\mathcal{D}$ if and only if $\mathrm{D}$ is an destroyer of $\mathcal{D}_{<\mathrm{r}}$ and $\mathcal{D}_{<\mathrm{r}}$ is Helly.

See Figure 2.1 for an example of a destroyer and the smallest destroyer. Har-Peled et al. first noticed that if $\mathcal{C} \subseteq \mathcal{D}$ is Helly and $D$ is a destroyer of $\mathcal{C}$, then the point $v \in \cap \mathcal{C}$ with minimum distance to $\mathrm{D}$ is unique. The distance between a point $v$ and a disk $\mathrm{D}$ is defined as the shortest distance between the point and any point that lies within or on the boundary of the disk D. They proved the geometric observation using contradiction. The unique point $v \in \cap \mathcal{C}$ is called 


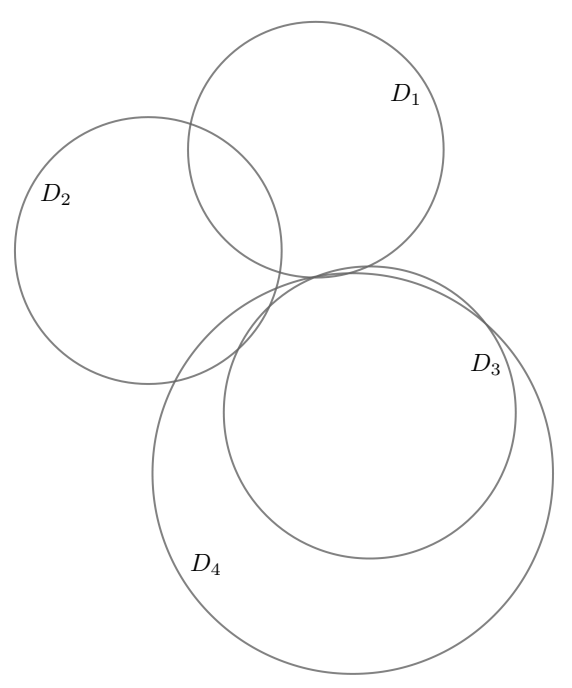

Figure 2.1: $D_{3}$ and $D_{4}$ are two destroyers of the Helly set $\left\{D_{1}, D_{2}\right\}$, and $D_{3}$ is the smallest destroyer of the set $\left\{D_{1}, D_{2}, D_{3}, D_{4}\right\}$

the extreme point for $\mathcal{C}$ and $D$, and we use $d(\cap \mathcal{C}, D)$ to represent the distance between the extreme point $v$ and the smallest destroyer D. Then, they proved the following geometric observation:

LEMMA 2.8. (Lemma 4.3 in [15]) Let $\mathcal{C}_{1} \subseteq \mathcal{C}_{2} \subseteq \mathcal{D}$ be two Helly sets and $\mathrm{D}$ be a destroyer of $\mathrm{C}_{1}$. Then we have that $\mathrm{d}\left(\cap \mathrm{C}_{1}, \mathrm{D}\right) \leqslant \mathrm{d}\left(\cap \mathrm{C}_{2}, \mathrm{D}\right)$. In particular, if the extreme point for $\mathrm{C}_{1}$ and $\mathrm{D}$ lies in $\cap \mathrm{e}_{2}$, then it is also the extreme point for $\mathcal{C}_{2}$ and $\mathrm{D}$. Otherwise, $\mathrm{d}\left(\cap \mathrm{C}_{1}, \mathrm{D}\right)<\mathrm{d}\left(\cap \mathrm{C}_{2}, \mathrm{D}\right)$.

Let $\mathcal{C}$ be a subset of $\mathcal{D}$ and $\mathrm{D}$ be the smallest destroyer of $\mathcal{C}$. They write $\operatorname{rad}(\mathcal{C})$ for the radius of $\mathrm{D}$ and $\operatorname{dist}(\mathcal{C})$ for the distance between $\mathrm{D}$ and the set $\cap \mathcal{C}_{<\operatorname{rad}(\mathcal{C})}$. If $\mathcal{C}$ is Helly, then $\operatorname{rad}(\mathcal{C})=\infty$, otherwise, $\operatorname{rad}(\mathcal{C})<\infty$. Given these notations, now define the weight of $\mathcal{C}$ as $w(\mathcal{C})=(\operatorname{rad}(\mathcal{C}),-\operatorname{dist}(\mathcal{C}))$, and let $\leqslant$ represent the lexicographic order on $\mathbb{R}^{2}$. Har-Peled et al. [15] proved that ( $\left.\mathcal{D}, w, \leqslant\right)$ is LP-type. They first showed that the three LP-type axioms hold for $(\mathcal{D}, w, \leqslant)$ using three lemmas. Then they designed the following violation test for $(\mathcal{D}, w, \leqslant)$ : given a set $\mathcal{B} \subseteq \mathcal{D}$ and a disk $E \in \mathcal{D}$ with radius $r$, determine (i) whether $\mathcal{B}$ is a basis for some subset of $\mathcal{D}$, and (ii) whether $E$ violates $\mathcal{B}$, i.e., whether $w(\mathcal{B} \cup\{\mathrm{E}\})<w(\mathcal{B})$ using the following strategy:

- If (i) $|\mathcal{B}|>3$; or (ii) $|\mathcal{B}|=3$ and $\mathcal{B}$ is Helly; or (iii) $|\mathcal{B}|=2$ and the $y$-minimum of $\cap \mathcal{B}$ is also the $y$-minimum of a single disk of $\mathcal{B}$, return "B is not a basis".

- If $|\mathcal{B}|=1$, then, if the $y$-minimum in $E \cap\{\cap \mathcal{B}\}$ differs from the $y$-minimum in $\cap \mathcal{B}$, return " $E$ violates $\mathcal{B}$ "; otherwise, return " $E$ does not violate $\mathcal{B}^{\prime \prime}$.

- If $\mathcal{B}=\left\{D_{1}, D_{2}\right\}$, find the $y$-minimum $v$ of $D_{1} \cap D_{2}$ and return "E violates $\mathcal{B}$ " if $v \notin E$, and " $E$ does not violate $\mathcal{B}$ ", otherwise. 
- Finally, assume that $\mathcal{B}=\left\{D_{1}, D_{1}, D_{2}\right\}$ is non-Helly with the smallest destroyer $D$. Let $r=\operatorname{rad}(\mathcal{B})$ be the radius of $D$ and $r^{\prime}$ be the radius of $E$ :

- If $r^{\prime}>r$, return "E does not violate $\mathcal{B}^{\prime}$.

- If $r^{\prime}<r$, find the vertex $v$ of $D_{1} \cap D_{2}$ that minimizes the distance to $E$ and return " $E$ violates $\mathcal{B}$ " if $v \notin E$, and “ $E$ does not violate $\mathcal{B}^{\prime \prime}$, otherwise.

The violation test clearly takes constant time. But in order to apply the algorithm by Chazelle and Matoušek [6], they still need to show that the range space $(\mathcal{D},\{v i o(\mathcal{B}) \mid \mathcal{B}$ is a basis of a subset in $\mathcal{D}\})$ has bounded VC dimension.

LEMMA 2.9. (Lemma 4.7 in [15]) The range space $(\mathcal{D},\{v i o(\mathcal{B}) \mid \mathcal{B}$ is a basis of a subset in $\mathcal{D}\}$ ) has VC-dimension at most 3.

Since $(\mathcal{D}, w, \leqslant)$ is LP-type and it satisfies all requirements of using the algorithm by Chazelle and Matoušek [6], so in deterministic linear time we can compute a basis for $\mathcal{D}$. If $\mathcal{D}$ is non-Helly, then the returned basis contains 3 disks and the largest disk of these 3 disks is the smallest destroyer of $\mathcal{D}$ [15].

LEMMA 2.10. (Lemma 4.8 in [15]) Given a set $\mathcal{D}$ of $n$ pairwise intersecting disks in the plane, we can decide in $\mathrm{O}(\mathrm{n})$ deterministic time whether $\mathcal{D}$ is Helly. If so, we can compute a point in $\cap \mathcal{D}$ in $\mathrm{O}(\mathrm{n})$ deterministic time. If not, we can compute the smallest destroyer $\mathrm{D}$ of $\mathcal{D}$ and two disks $\mathrm{E}, \mathrm{F} \in \mathcal{D}<\mathrm{r}$ that form a non-Helly triple with $\mathrm{D}$. Here, $\mathrm{r}$ is the radius of $\mathrm{D}$.

\subsection{EXISTING ALGORITHMS}

In this section, we will discuss the existing linear-time algorithms for piercing pairwise intersecting disks.

Hadwiger and Debrunner [13] showed that any set of pairwise intersecting unit disks can be pierced by 3 points. Their construction is based on a regular hexagon with sides of length $\frac{2}{\sqrt{3}}$ that covers the centers of all the disks in the set. Let the vertices of the hexagon be $\left\{v_{1}, v_{2}, \ldots, v_{6}\right\}$. Then they claimed that the middle points of the edges of the triangle $\Delta v_{1} v_{3} v_{5}$ pierce the given set of unit disks.

The two algorithms for piercing pairwise intersecting arbitrary disks both use LP-type problems as a subroutine. Let $\mathcal{D}$ be a set of pairwise intersecting disks of arbitrary radius. The algorithm by Har-Peled et al. computes the 5 piercing points for $\mathcal{D}$ based on the smallest destroyer of $\mathcal{D}$. By lemma 2.4, a piercing point can be computed for a Helly set in linear time, so they assumed $\mathcal{D}$ is non-Helly. Let $\{D, E, F\}$ be the three disks as defined in the statement of lemma 2.10. Among these three disks, they first find two disks whose lens angle is at least $120^{\circ}$. Lens angle is defined in [15]: 


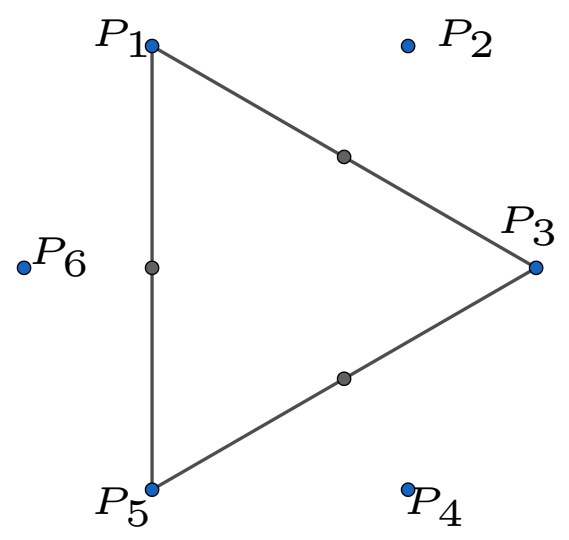

Figure 2.2: If $\left\{v_{1}, v_{2}, \ldots, v_{6}\right\}$ contains the centers of all the unit disks, then the middle points of the edges of the triangle $\Delta v_{1} v_{3} v_{5}$ pierce the set of disks.

DEFINITION 2.11 (Lens angle). Let $\mathrm{D}_{1}$ and $\mathrm{D}_{2}$ be two intersecting disks in the plane with center $\mathrm{c}_{1}$ and $\mathrm{c}_{2}$, respectively. Let one of the intersection points between the boundaries of these two disks be $u$. The lens angle between $\mathrm{D}_{1}$ and $\mathrm{D}_{2}$ is defined as $\angle \mathrm{c}_{1} \mathrm{uc}_{2}$.

For convenience, we call the two disks with lens angle at least $120^{\circ}$ $D_{1}$ and $D_{2}$. Let their centers be $c_{1}$ and $c_{2}$, and let their radius be $r_{1}$ and $r_{2}$. By relabelling, we can assume that $r_{1} \geqslant r_{2}$. We first increase $r_{2}$ while keeping $c_{2}$ fixed until $r_{2}=r_{1}$. Then we move $c_{2}$ along the line spanned by $c_{1}$ and $c_{2}$ until the lens angle between $D_{1}$ and $D_{2}$ becomes exactly $120^{\circ}$. By a proper scaling, rotation, and translation, we assume the midpoint of the line segment $c_{1} c_{2}$ is the origin, $r_{1}=$ $r_{2}=1$, and both $c_{1}$ and $c_{2}$ lie on the $x$-axis. Har-Peled et al. then proved that $\left\{c_{1}, c_{2},(0,1),(0,-1)\right\}$ pierce any disk with radius $\geqslant 1$ and intersects both $D_{1}$ and $D_{2}$. Therefore, these four points pierce $\mathcal{D}_{\geqslant 1}$. Since $D$ is the largest disk of $\{D, E, F\}$, so $r \geqslant 1$. Therefore, these four points pierce $\mathcal{D}_{\geqslant r}$. Since $D$ is the smallest destroyer of $\mathcal{D}, \mathcal{D}_{<r}$ is Helly and we can apply lemma 2.10 to find a point that pierces $\mathcal{D}_{<r}$. These steps all take linear time.

The algorithm proposed by Carmi et al. [3] depends on a different, slightly simpler variant of the LP-type problem. All the coordinates of the piercing points are computed with respect to the smallest disk that intersects every disk in the set. Let $\mathcal{D}$ be the set of disks and let $d^{*}$ be the smallest disk that intersects every disk in $\mathcal{D}$. They find 4 points that pierce $\mathcal{D} \cup\left\{\mathrm{d}^{*}\right\}$. Let $\mathrm{c}^{*}$ be the center of $\mathrm{d}^{*}$ and let its radius be 1 . Let $\mathcal{D}^{-}$be the set of disks in $\mathcal{D}$ that do not contain $c^{*}$. Let $d_{\text {min }}$ be the smallest disk in $\mathcal{D}^{-}$and let $r_{\min }$ be its radius. $r_{\min } \geqslant 1$. Their algorithm distinguishes between three main cases, depending on the value of $r_{\min }$. When $r_{\min } \geqslant 4$, then there exist 3 points that pierce all the disks in $\mathcal{D}^{-}$, together with $\mathrm{c}^{*}$ we have four points that pierce $\mathcal{D}$. When $r_{\min } \leqslant 2$, they further split the case into three subcases. 
In each subcase, they suggest one candidate point set consisting of 4 points that pierce $\mathcal{D}$. When $2<r_{\min }<4$, they consider four different subcases. In each subcase, there exist a set of 4 points that pierce $\mathcal{D}$. Therefore, they suggested 8 different candidate point sets, and they proved that one of these candidate sets pierces $\mathcal{D}$. The computation of $\mathrm{d}^{*}$ takes linear time as it is an LP-type problem. The computation of all the piercing points also takes linear time, so their algorithm runs in linear time.

Even though these two algorithms can compute a set of points that pierce the given set of arbitrary disks in deterministic linear time, they use the heavy machinery of the LP-type framework. We propose algorithms that use some fundamental geometric properties and avoid using the LP-type framework. In turn, we feel that our algorithms are simpler and easier to implement.

\subsection{PIERCING EIGHT DISKS WITH THREE POINTS}

In this section, we will prove that any set of 8 pairwise intersecting arbitrary disks can be pierced by 3 points. Before we come to the proof, we first present a useful geometric observation.

LEMMA 2.12. Every set of 4 pairwise intersecting disks whose centers are in convex position contains a Helly triple.

Proof. Let $a, b, c$, and $d$ be the centers of these disks in the order along their convex hull. Let $\mathrm{D}(\mathrm{p})$ be the disk centered at point $p$. Let $x$ be a point on line segment ac that lies in the intersection of $D(a)$ and $D(c)$; $x$ splits ac into two line segments, $a x$ and $x c$. Let $y$ be a point on line segment bd that lies in the intersection of $D(b)$ and $D(d)$; $y$ splits bd into by and $y d$. Among these four line segments, two of them must cross. With a suitable translation and relabeling, assume ax and by intersects at point $\mathrm{o}$. By applying the Triangle Inequality on $\triangle$ box and $\triangle a o y,|a x|+|b y|>|a y|+|b x|$. This implies that either ax is larger than ay or by is larger than bx. In the former case, $|a x|>|a y|$ implies that $y$ lies within $D(a)$, so $y \in D(a) \cap D(b) \cap D(d)$. In the latter case, using a similar argument, we can conclude that $x \in D(a) \cap D(b) \cap$ $\mathrm{D}(\mathrm{c})$. Figure 2.3 shows the latter case and $\{\mathrm{D}(\mathrm{a}), \mathrm{D}(\mathrm{b}), \mathrm{D}(\mathrm{c})\}$ is a Helly triple.

By the Happy Ending problem [10], it is known that every set of 5 points contains 4 points that are in convex position. We can then apply lemma 2.12 to prove the following theorem:

THEOREM 2.13. Every set of 8 pairwise intersecting arbitrary disks can be pierced by at most 3 points.

Proof. We can first find 4 points that are in convex position out of these 8 centers. Then by lemma 2.12, the 4 disks centered at these 


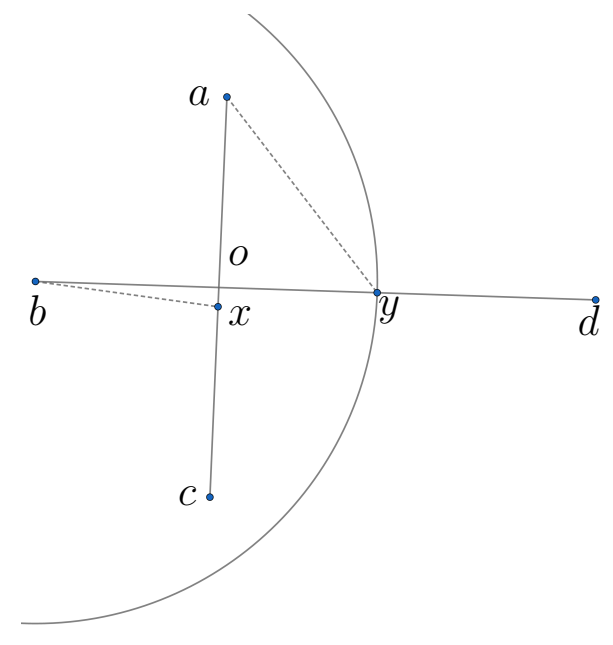

Figure 2.3: If $a x$ and by intersects and $|b y|>|b x|$, then $x$ lies within $D(b)$ and the intersection of $\mathrm{D}(\mathrm{a}), \mathrm{D}(\mathrm{b})$, and $\mathrm{D}(\mathrm{c})$ is nonempty.

points contain a Helly triple. Let $p_{1}$ be a point that lies in the common intersection of this Helly triple. Despite the three disks in the Helly triple, there are 5 more disks that may not be pierced by $p_{1}$. We can again find 4 disks whose centers are in convex position by the Happy Ending problem. Lemma 2.12 states that we can find a new Helly triple among these 4 disks and we let $p_{2}$ be a point that lies in the intersection of this new Helly triple. Lastly, we choose $p_{3}$ to be a point that lies in the common intersection of the two remaining disks.

Danzer [8] had given a construction of 10 pairwise intersecting arbitrary disks that cannot be pierced by 3 points. But it is still unknown whether any set of 9 pairwise intersecting arbitrary disks can be pierced by 3 points or not. 
In this chapter, we present a deterministic linear-time algorithm for piercing any set of pairwise intersecting unit disks with 3 points. The algorithm uses simple geometric transformation on the given set of disks and does not require solving any LP-type problem. This algorithm is introduced and analyzed in Section 3.I. In addition, we propose another algorithm that requires the knowledge of the smallest disk that intersects every disk in the set. We have shown in Section 2.2 that such disk can be computed in deterministic linear time. The algorithm is introduced in Section 3.2. At the end of this chapter, in Section 3.3, we present a lower bound construction consisting of 9 pairwise intersecting unit disks that cannot be pierced by 2 points.

Let $\mathcal{D}$ be a set of pairwise intersecting unit disks; each disk $D_{i} \in \mathcal{D}$ is described by its radius $r_{i}$ and its center $c_{i}=\left(x_{i}, y_{i}\right)$. We denote the Euclidean distance between points $a$ and $b$ by $|a b|$.

\subsection{ALgORITHM Without SOLVING ANY LP-TYPE PROBLEM}

Given a set of pairwise intersecting unit disks, the existing argument that proves the existence of 3 piercing points requires the knowledge of the regular hexagon that covers the centers of all the disks [13]. We will see in the following theorem that we can find the piercing points without finding the hexagon.

THEOREM 3.1. Let $\mathcal{D}$ be a set of pairwise intersecting unit disks. In $\mathrm{O}(|\mathcal{D}|)$ time, we can compute 3 points that pierce $\mathcal{D}$.

Proof. If $|\mathcal{D}| \leqslant 4$, then it is obvious how we can pierce $\mathcal{D}$ with 2 points. So we assume $|\mathcal{D}|>4$. Let $D_{1}$ be a disk in $\mathcal{D}$. We reduce $r_{1}$ while keeping $c_{1}$ fixed until $D_{1}$ is tangent to a second disk $D_{2} \in \mathcal{D}$. This can be completed in linear time by computing the distance from $c_{1}$ to all other disks in $\mathcal{D}$. After shrinking, $D_{1}$ has radius $r_{1} \leqslant 1$, and it is tangent to $D_{2}$. By a proper translation and rotation, we move $c_{1}$ to the origin and $c_{2}$ to a point that lies on the positive $y$-axis with coordinate $\left(0, r_{1}+1\right)$. Let $D_{0}$ be a unit disk with center $c_{0}=\left(0, r_{1}-1\right)$. Since $r_{1} \leqslant 1, D_{1} \subseteq D_{0}$. Any disk that intersects $D_{1}$ also intersects $D_{0}$. Let $D_{1}^{\prime}$ and $D_{2}^{\prime}$ be two disks with radius 2 and centers $c_{0}$ and $c_{2}$, respectively; see Figure 3.1. If a unit disk $D_{i}$ intersects $D_{0}$ and $D_{2}$, then $\left|c_{0} c_{i}\right| \leqslant 2,\left|c_{2} c_{i}\right| \leqslant 2$ and $c_{i} \in D_{1}^{\prime} \cap D_{2}^{\prime}$. 


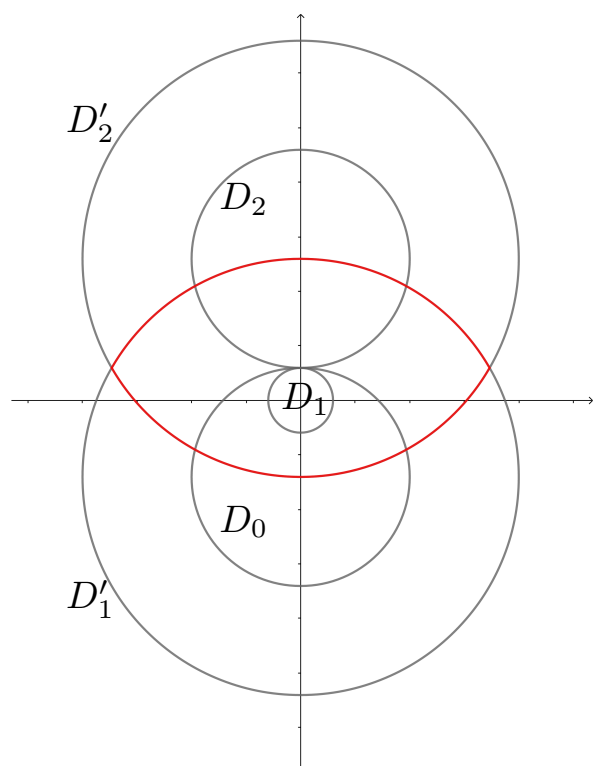

Figure 3.1: Configuration of Theorem 3.1.

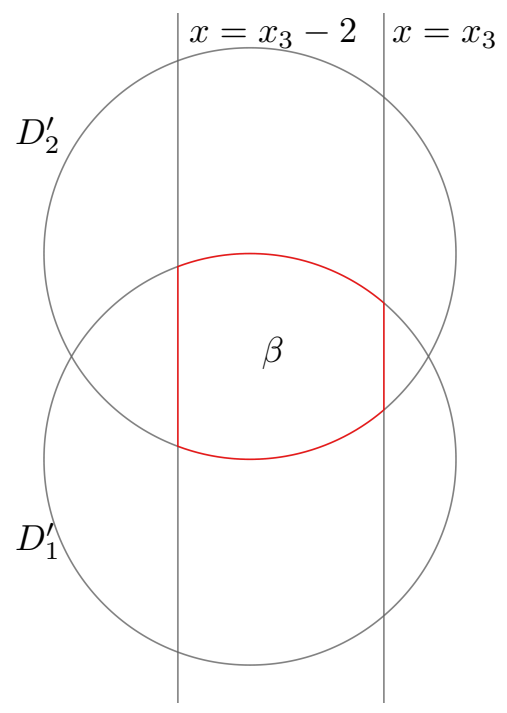

Figure 3.2: $\beta$ is the region that needs to be covered.

Let $\mathrm{D}_{3}$ be the disk in $\mathcal{D}$ with the maximum $x$-coordinate. Since it must intersect $D_{1}$ and $D_{2}$, we note that $0 \leqslant x_{3} \leqslant \sqrt{3}$. $D_{3}$ can be found in linear time. For every disk $D_{i} \in \mathcal{D},\left|c_{i} c_{3}\right| \leqslant 2$ since $D_{i}$ and $D_{3}$ intersect. We can conclude that $\left|x_{i} x_{3}\right| \leqslant 2$ since both $D_{i}$ and $\mathrm{D}_{3}$ are unit disks. Therefore, in addition to being in $D_{1}^{\prime} \cap \mathrm{D}_{2}^{\prime}$, the $x$-coordinates of all the centers lie in the interval $\left[x_{3}-2, x_{3}\right]$. Let $\beta$ represent the region where all the centers of the disks in $\mathcal{D}$ must lie as illustrated in red in Fig 3.2. We say an area is covered by a point set $P$ if every point in the area has distance at most 1 to at least 1 point in $P$. Therefore, if we can find 3 points that cover $\beta$, then those three points pierce every disk in $\mathcal{D}$. As noted above, we have that 


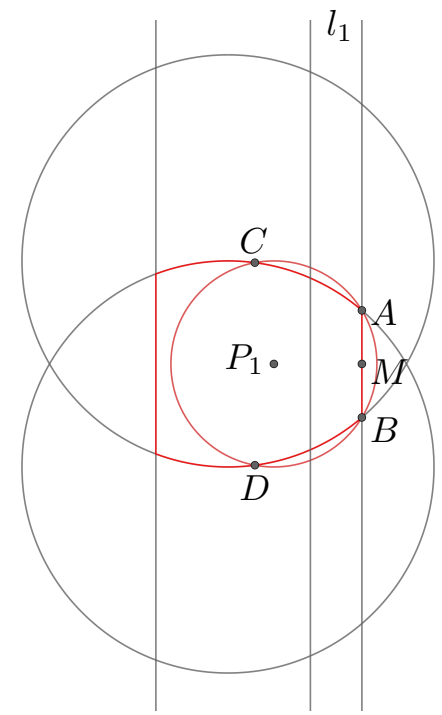

Figure 3.3: How to choose $P_{1}$.

$0 \leqslant x_{3} \leqslant \sqrt{3}$. We consider two cases, namely when $1 \leqslant x_{3} \leqslant \sqrt{3}$ and $0 \leqslant x_{3}<1$.

Case 1: $1 \leqslant x_{3} \leqslant \sqrt{3}$. Let $A$ (resp. B) be the rightmost point of $\beta$ on the boundary of $D_{1}^{\prime}$ (resp. $D_{2}^{\prime}$ ). The first point $P_{1}$ is chosen to be a point that falls in $\beta$ and has distance 1 to both $A$ and $B$. Let $C_{1}$ be a circle of radius 1 centered at $P_{1}$. See Figure 3.3.

Let $l_{1}$ be the vertical line $x=x_{3}-\frac{1}{2}$. First, we prove that $P_{1}$ always lies to the left of $l_{1}$. Let the midpoint of line segment $A B$ be $M .|A B|$ decreases as $x_{3}$ increases and it is maximized when $x_{3}=1$. So $|A B| \leqslant$ $\sqrt{3}$ and $|A M| \leqslant \frac{\sqrt{3}}{2}$. Since $\triangle P_{1} A M$ is a right triangle and $\left|A P_{1}\right|=1$, by the Pythagorean theorem, $\left|P_{1} M\right| \geqslant \frac{1}{2}$. Therefore, $P_{1}$ always lies to the left of $l_{1}$. Let the intersection point of circle $C_{1}$ and $D_{1}^{\prime}$ different from $A$ be labeled $C$, and the intersection point of circle $C_{1}$ and $D_{2}^{\prime}$ different from $B$ be labeled $D$. Since the circle $C_{1}$ is tangent to both $y=r_{1}+1$ line and $y=r_{1}-1$ line, both $C$ and $D$ lie to the left of $P_{1}$. See Figure 3.3. Since the radius of $C_{1}$ is 1 , the radius of $D_{1}^{\prime}$ is 2 , and $C$ lies to the left of $l_{1}$, we have that the clockwise arc from $C$ to $A$ on the boundary of $D_{1}^{\prime}$ and the clockwise arc from $B$ to $D$ on the boundary of $D_{2}^{\prime}$ are both contained in $C_{1}$. Therefore, the center of any unit disk of $\mathcal{D}$ that lies on or to the right of $l_{1}$ is contained in the disk $C_{1}$. We now show how to compute points $P_{2}$ and $P_{3}$ to pierce all the disks that do not contain $P_{1}$, namely the disks in $\mathcal{D}$ whose centers are in $\beta$ but outside disk $C_{1}$. The coordinates of $A, B, P_{1}, P_{2}, P_{3}$ are given in Section 3.1.1.

Now consider the rectangle formed by the following 4 points: $E=$ $\left(x_{3}-\frac{1}{2}, r_{1}+1\right), F=\left(x_{3}-\frac{1}{2}, r_{1}-1\right), G=\left(x_{3}-2, r_{1}+1\right), H=\left(x_{3}-\right.$ $\left.2, r_{1}-1\right)$. See Fig 3.4. Since $D_{1}^{\prime}$ is tangent to the line $y=r_{1}+1$ at $(0, r+1)$, and $D_{2}^{\prime}$ is tangent to the line $y=r_{1}-1$ at $(0, r-1)$, the area $\beta \cap\left\{x<x_{3}-\frac{1}{2}\right\}$ as shown in Fig 3.4 is contained completely within 


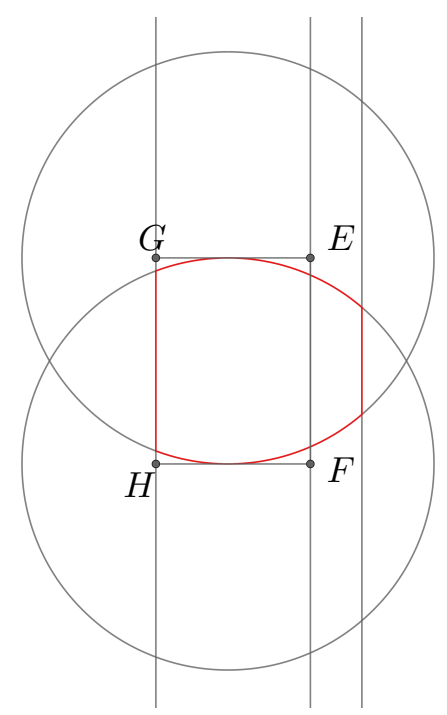

Figure 3.4: Remaining area to be covered.

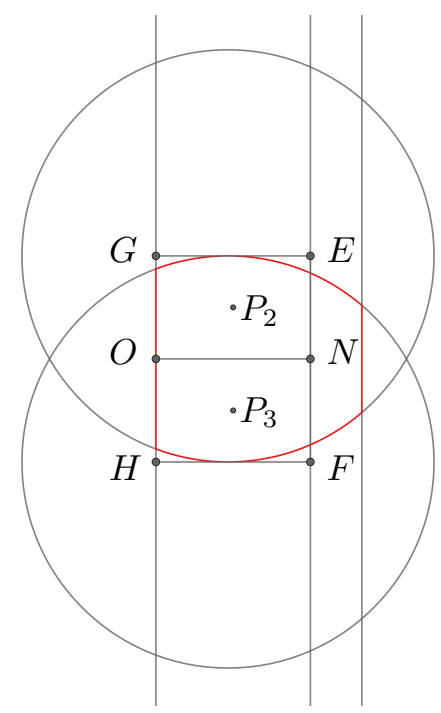

Figure 3.5: How to choose $P_{2}$ and $P_{3}$.

the rectangle EFHG. If the points $P_{2}$ and $P_{3}$ cover this rectangle, then we are done. Let $\mathrm{N}$ be the midpoint of line segment $\mathrm{EF}$ and let $\mathrm{O}$ be the midpoint of line segment GH. See Fig 3.5. We choose $P_{2}$ to be the center of the rectangle ENOG. $|\mathrm{EN}|=1$ and $|\mathrm{NO}|=\frac{3}{2}$, by the Pythagorean Theorem, $\mathrm{P}_{2}$ 's distance to all four vertices of the rectangle is $\frac{\sqrt{13}}{4}$. Therefore, if a unit disk's center falls in the rectangle ENOG, then the disk is pierced by $P_{2}$. Symmetrically pick $P_{3}$ to be the center of the rectangle NFHO. Then any unit disk in $\mathcal{D}$ whose center falls to the left of $l_{1}$ is pierced by one of $P_{2}$ and $P_{3}$.

Case 2: $0 \leqslant x_{3}<1$. In this case, $x_{3}-2<-1$ and $\left|x_{3}-2\right|>1$. The vertical line on the left is $x=x_{3}-2$, and the vertical line on the right is $x=x_{3}$. If we reflect all the disks in $\mathcal{D}$ about the $y$-axis, the vertical line on the right becomes $x=\left|x_{3}-2\right|$, and we know $\left|x_{3}-2\right|>1$. Then 
let $x_{3}^{\prime}=\left|x_{3}-2\right|$, and we compute the piercing points using $x=x_{3}^{\prime}$ and $x=x_{3}^{\prime}-2$ as in Case 1. Then the three computed points pierce D.

\subsubsection{Coordinates of Points in Theorem 3.1}

Recall that $r_{1}$ is the radius of $D_{1}$ and $r_{1} \leqslant 1, x_{3}$ is the $x$-coordinate of the center of the rightmost disk in $\mathcal{D}$.

The coordinate of $A$ is $\left(x_{3}, \sqrt{4-x_{3}^{2}}+r_{1}-1\right)$.

The coordinate of $B$ is $\left(x_{3},-\sqrt{4-x_{3}^{2}}+r_{1}+1\right)$.

The coordinate of $P_{1}$ is $\left(x_{3}-\sqrt{2 \sqrt{4-x_{3}^{2}}+x_{3}^{2}-4}, r_{1}\right)$.

The coordinate of $P_{2}$ is $\left(x_{3}-\frac{5}{4}, r_{1}+\frac{1}{2}\right)$.

The coordinate of $P_{3}$ is $\left(x_{3}-\frac{5}{4}, r_{1}-\frac{1}{2}\right)$.

\subsection{ALGORITHM WITH SOLVING AN LP-TYPE PROBLEM}

We have just shown how we can pierce $\mathcal{D}$ in deterministic linear time without solving any LP-type problem. In this section, we are going to present an algorithm for computing the three piercing points with respect to the smallest disk that intersects every disk in $\mathcal{D}$. The algorithm is straightforward with the help of the following geometric observation:

LEMMA 3.2. The smallest disk that is tangent to three pairwise intersecting unit disks that are non-Helly has radius at most $\frac{2}{\sqrt{3}}-1$.

Proof. Let $\left\{\mathrm{D}_{1}, \mathrm{D}_{2}, \mathrm{D}_{3}\right\}$ be three pairwise intersecting unit disks that are non-Helly and let $\mathrm{D}$ be the smallest disk that is tangent to these three disks. Let $\mathrm{c}$ be the center of $\mathrm{D}$ and let $\mathrm{r}$ be its radius. $\angle \mathrm{c}_{1} \mathrm{cc}_{2}+$ $\angle c_{2} c_{3}+\angle c_{1} c_{3}=360^{\circ}$, so there must exist an angle that is at least $120^{\circ}$. Without loss of generality, assume $\angle c_{1} c_{2} \geqslant 120^{\circ} .\left|c_{1} c\right|=$ $\left|c_{2} c\right|=1+r$, so $\left|c_{1} c_{2}\right| \geqslant \sqrt{3}(1+r)$. Since $D_{1}$ and $D_{2}$ are two unit disks and they intersect, so $\left|c_{1} c_{2}\right| \leqslant 2$. $\sqrt{3}(1+r) \leqslant 2$. By rearranging terms in the equation, we have that $r \leqslant \frac{2}{\sqrt{3}}-1$.

Using lemma 3.2, we can give an alternate proof for Theorem 3.1.

Proof. We can determine if the given set of disks is Helly or not using the technique outlined in the proof of lemma 2.10. If $\mathcal{D}$ is Helly, then a piercing point is returned by the algorithm. We assume that $\mathcal{D}$ is nonHelly. It is known that the smallest disk that intersects every disk in $\mathcal{D}$ can be computed in deterministic linear time [19]. Let $\mathrm{D}$ be the smallest disk that intersects every disk in $\mathcal{D}$, and let $\mathrm{c}$ and $r$ represent its center and radius, respectively. Our choice of $D$ ensures that $D$ is tangent to three disks in $\mathcal{D}$; otherwise, the radius of $\mathrm{D}$ can be reduced 


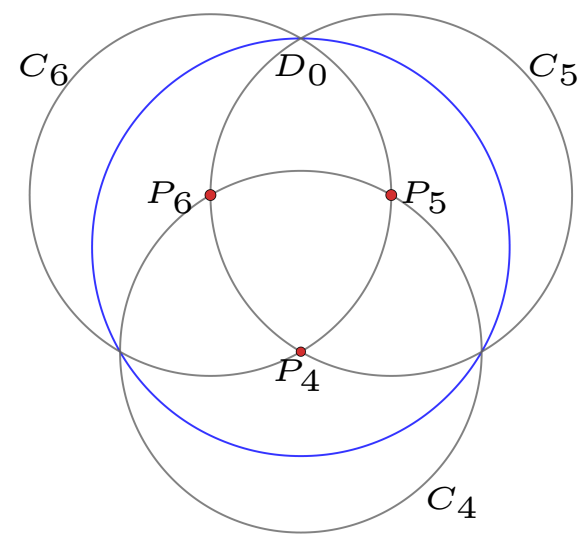

Figure 3.6: The location of $P_{4}, P_{5}$ and $P_{6}$ and how $D_{0}$ is covered by $C_{4}, C_{5}$ and $\mathrm{C}_{6}$.

which contradicts minimality. These three disks are non-Helly. Therefore, by lemma $3.2, r \leqslant \frac{2}{\sqrt{3}}-1$. Every disk $D_{i} \in \mathcal{D}$ with center $c_{i}$ intersects $D$, so $\left|c_{i} c\right| \leqslant \frac{2}{\sqrt{3}}$. Let $D_{0}$ be a disk centered at $c$ with radius $\frac{2}{\sqrt{3}}$. By translation, we make $c$ the origin. The center of all the disks in $\mathcal{D}$ falls in $D_{0}$. Let $P_{4}=\left(0,-\frac{1}{\sqrt{3}}\right), P_{5}=\left(\frac{1}{2}, \frac{1}{2 \sqrt{3}}\right), P_{6}=\left(-\frac{1}{2}, \frac{1}{2 \sqrt{3}}\right)$. Let $C_{4}, C_{5}$, and $C_{6}$ be three circles of radius 1 and centers $P_{4}, P_{5}$ and $\mathrm{P}_{6}$, respectively; see Figure 3.6.

Let $A$ (resp. B) be the intersection point between $C_{4}$ and $D_{0}$ that falls in the third (resp. fourth) quadrant. Any unit disk whose center falls in the $C_{4} \cap D_{0}$ is pierced by $P_{4}$. This region is illustrated in red in Figure 3.7. Now we want to prove that $P_{5}$ and $P_{6}$ pierce $D_{0} \backslash C_{4}$. Let $C_{5}$ 's intersection point with $D_{0}$ other than $B$ be $C$, so the area illustrated in green in Figure 3.7 is covered by $P_{5}$. The red bounded area and the green bounded area intersects at two points, one is $B$, and the other one is $P_{6} . P_{6}$ has distance 1 to both point $A$ and point C. So the area $C_{0} \backslash\left\{C_{4} \cup C_{5}\right\}$ is covered by $P_{6}$.

\subsection{LOWER BOUND CONSTRUCTION}

We now present a set of 9 pairwise intersecting unit disks that cannot be pierced by 2 points. See Figure 3.8 for the positioning of the disks being constructed in Theorem 3.3.

THEOREM 3.3. There exists a set of 9 pairwise intersecting unit disks that cannot be pierced by 2 points.

Proof. We begin the construction by placing 3 unit disks $\mathrm{D}_{1}, \mathrm{D}_{2}, \mathrm{D}_{3}$ centered at $(0,0),(2,0),(1, \sqrt{3})$ respectively. These points are the vertices of an equilateral triangle with side length 2 . Denote the center of $D_{i}$ by $c_{i} . D_{1}, D_{2}$ and $D_{3}$ are all tangent to each other. Let $C_{1}, C_{2}$, and $C_{3}$ be three disks of radius 2 centered at $(0,0),(2,0)$ and $(1, \sqrt{3})$, 


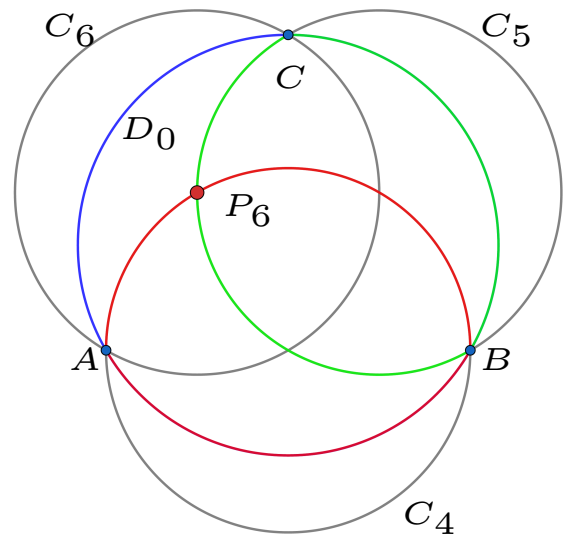

Figure 3.7: $P_{4}, P_{5}$ and $P_{6}$ cover $D_{0}$.

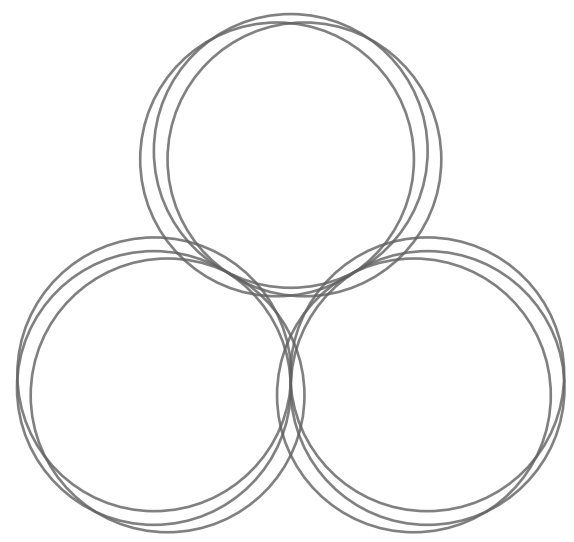

Figure 3.8: A set of 9 pairwise intersecting unit disks that cannot be pierced by 2 points.

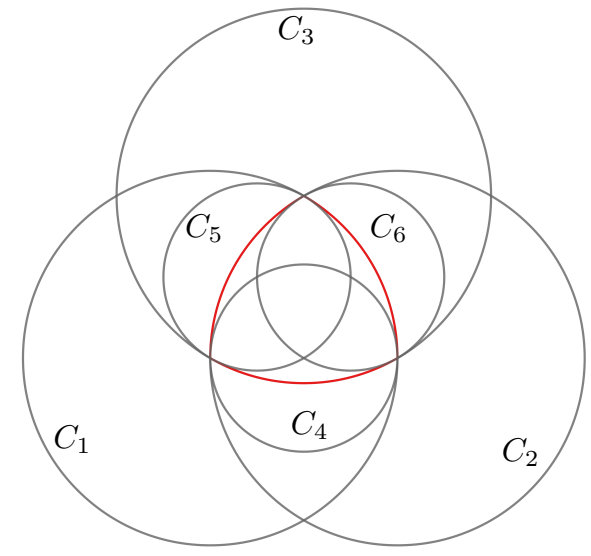

Figure 3.9: The position of $\mathrm{C}_{1}, \mathrm{C}_{2}, \mathrm{C}_{3}, \mathrm{C}_{4}, \mathrm{C}_{5}, \mathrm{C}_{6}$. 
respectively. If a unit disk $D_{i}$ with center $c_{i}$ intersects $D_{1}, D_{2}$, and $D_{3}$, then $c_{i} \in C_{1} \cap C_{2} \cap C_{3}$. The reuleaux triangle formed by $C_{1}, C_{2}$, and $C_{3}$ is illustrated in red in Figure 3.9. let $C_{4}, C_{5}$, and $C_{6}$ be three disks of radius 1 centered at $(1,0),\left(\frac{1}{2}, \frac{\sqrt{3}}{2}\right)$, and $\left(\frac{3}{2}, \frac{\sqrt{3}}{2}\right)$, respectively. We then create 6 more unit disks as follows:

- We create $D_{1}^{\prime}$ with center $c_{1}^{\prime}=\left(2-\sqrt{4-\epsilon^{2}}, \epsilon\right)$. $c_{1}^{\prime}$ lies on the boundary of $\mathrm{C}_{2}$ but falls outside of $\mathrm{C}_{4}$.

- We create $D_{1}^{\prime \prime}$ with center $c_{1}^{\prime \prime}=\left(\epsilon, \sqrt{3}-\sqrt{4-(\epsilon-1)^{2}}\right) . c_{1}^{\prime \prime}$ lies on the boundary of $C_{3}$ but falls outside of $C_{5}$.

- We create $\mathrm{D}_{2}^{\prime}$ with center $c_{2}^{\prime}=\left(2-\epsilon, \sqrt{3}-\sqrt{4-(\epsilon-1)^{2}}\right) \cdot c_{2}^{\prime}$ lies on the boundary of $C_{3}$ but falls outside of $C_{6}$.

- We create $D_{2}^{\prime \prime}$ with center $c_{2}^{\prime \prime}=\left(\sqrt{4-\epsilon^{2}}, \epsilon\right)$. $c_{2}^{\prime \prime}$ lies on the boundary of $\mathrm{C}_{1}$ but falls outside of $\mathrm{C}_{4}$.

- We create $D_{3}^{\prime}$ with center $c_{3}^{\prime}=\left(1+\epsilon, \sqrt{4-(1+\epsilon)^{2}}\right)$. $c_{3}^{\prime}$ lies on the boundary of $C_{1}$ but falls outside of $C_{5}$.

- We create $D_{3}^{\prime \prime}$ with center $c_{3}^{\prime \prime}=\left(1-\epsilon, \sqrt{4-(1+\epsilon)^{2}}\right)$. $c_{3}^{\prime \prime}$ lies on the boundary of $\mathrm{C}_{2}$ but falls outside of $\mathrm{C}_{6}$.

In the above construction, we assume $\epsilon=0.01$. See Fig 3.10 for the position of $c_{1}^{\prime}$ and $c_{1}^{\prime \prime}$. Now we want to show that these 9 disks pairwise intersect. We first prove that $\mathrm{D}_{1}^{\prime}$ intersects all the other 8 disks. $\left|c_{1}^{\prime} c_{1}\right| \approx 0.1,\left|c_{1}^{\prime} c_{1}^{\prime \prime}\right| \approx 0.18$, so $D_{1}^{\prime}$ intersects $D_{1}$ and $D_{1}^{\prime \prime}$. We prove $\mathrm{D}_{1}^{\prime}$ intersects the other 6 disks by showing that $c_{1}^{\prime}$ has distance $\leqslant 2$ to all the centers of these 6 disks. $c_{1}^{\prime}$ lies on the boundary of $C_{2}$, so $\left|c_{1}^{\prime} c_{2}\right|=2$. $c_{1}^{\prime}$ lies within $C_{3}$, so $\left|c_{1}^{\prime} c_{3}\right|<2$. Let $C_{7}$ be a circle of radius 2 centered at $c_{1}^{\prime}$. $C_{7}$ intersects $C_{1}$ at two points; one of them is $c_{2}$. The other intersection point lies to the left of $c_{3}$; See Figure 3.11. Therefore, $c_{3}^{\prime}, c_{3}^{\prime \prime}, c_{2}^{\prime}$, and $c_{2}^{\prime \prime}$ must lie within $P_{7}$. The unit disks centered at these points intersect $\mathrm{D}_{1}^{\prime}$. Using a similar argument, we can prove that any disk in this set intersects all other disks in the set. Therefore, we can conclude that these 9 disks are pairwise intersecting.

For the sake of contradiction, suppose $P=\left\{p_{1}, p_{2}\right\}$ pierces these 9 unit disks. Then there must exist a point $p_{1}$ that pierces at least 2 of $D_{1}, D_{2}$ and $D_{3}$. Without loss of generality, assume $p_{1}$ pierces $D_{1}$ and $D_{2}$. Then $p_{1}$ must be $(1,0)$ since $\left|c_{1} c_{2}\right|=2$. Based on our construction we know that $p_{1}$ does not pierce $D_{1}^{\prime}$ and $D_{2}^{\prime \prime}$. $p_{1}$ 's distances to $c_{3}, c_{3}^{\prime}$ and $c_{3}^{\prime \prime}$ are all greater than 1 , so $p_{1}$ does not pierce $D_{3}, D_{3}^{\prime}$ and $D_{3}^{\prime \prime}$. Thus, $\mathrm{p}_{2}$ pierces these 5 disks. In particular, $\mathrm{p}_{2}$ pierces $\mathrm{D}_{1}^{\prime}, \mathrm{D}_{2}^{\prime \prime}$ and $\mathrm{D}_{3}$, so $p_{2} \in D_{1}^{\prime} \cap D_{2}^{\prime \prime} \cap D_{3}$. But the circumscribed circle of $\triangle c_{1}^{\prime} c_{2}^{\prime \prime} c_{3}$ has radius 1.15, so these 3 disks are non-Helly. We need at least 2 points to pierce a non-Helly triple. Therefore, we have a contradiction which shows that the minimum number of points required to pierce these 9 disks is 3 . 


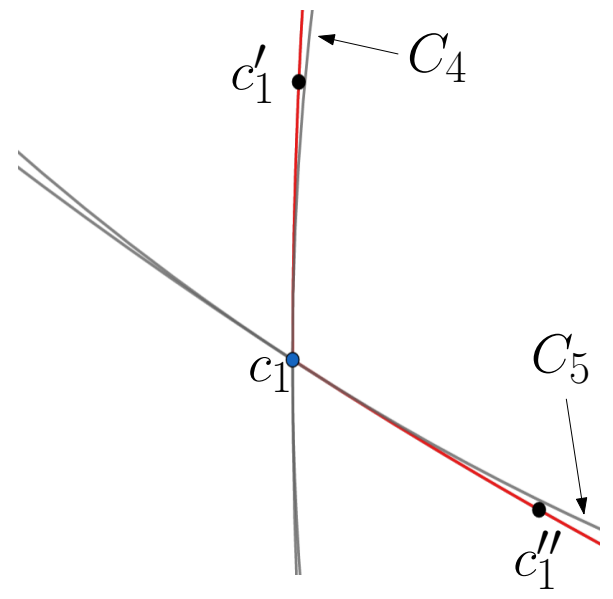

Figure 3.10: The position of $c_{1}^{\prime}$ and $c_{1}^{\prime \prime}$.

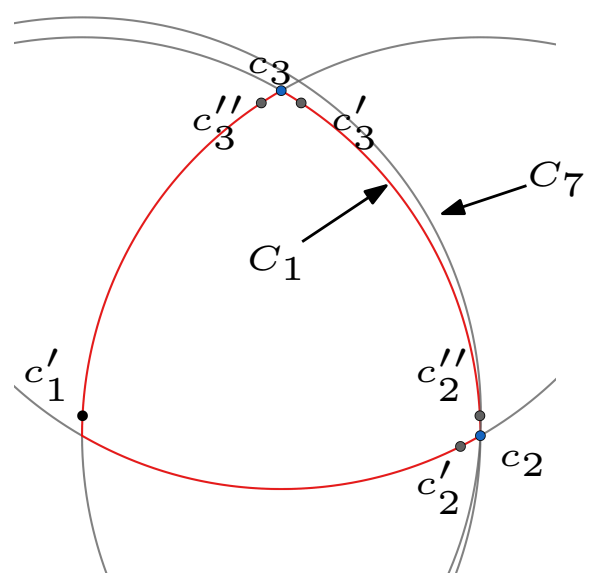

Figure 3.11: $\mathrm{D}_{1}^{\prime}$ intersects all other disks. 
PIERCING PAIRWISE INTERSECTING DISKS OF ARBITRARY RADIUS

In this chapter, we present a deterministic linear-time algorithm for piercing a set of pairwise intersecting disks of arbitrary radius. We first present an important result that inspired our work in Section 4.I. Then the algorithm is presented and analyzed in Section 4.2. Similar to the algorithm we presented in Section 3.1, our algorithm does not require solving any LP-type problem.

Let $\mathcal{D}$ be a set of pairwise intersecting arbitrary disks; each disk $D_{i} \in \mathcal{D}$ is described by its radius $r_{i}$ and its center $c_{i}=\left(x_{i}, y_{i}\right)$. We denote the Euclidean distance between points $a$ and $b$ by $|a b|$.

\section{I PIERCING WITH SEVEN POINTS}

Even though 4 points are always sufficient to pierce any set of pairwise intersecting disks [8], finding such 4 points is sometimes difficult. However, we can always find a piercing set of 7 points whose coordinates can be computed very easily.

The following theorem is first discovered and proved by Bose et al. [2]. In their proof, they compute the coordinates of these 7 points with respect to the smallest disk that intersects every disk in $\mathcal{D}$. The problem of finding such a disk is LP-type. However, we prove that we can find 7 points that pierce the given set of disks without involving any LP-type machinery.

THEOREM 4.1. (Theorem 2 in [2]) Given a set $\mathcal{D}$ of $m$ pairwise intersecting disks in the plane, 7 points are sufficient to pierce every disk.

Proof. Let $\mathrm{D}_{1} \in \mathcal{D}$ be the smallest disk in $\mathcal{D}$. Finding this disk takes linear time. By scaling and translation, we assume $D_{1}$ is centered at the origin and its radius is 1 . Let $P_{1}=(0,0), P_{2}=(\sqrt{3}, 0), P_{3}=$ $\left(\frac{\sqrt{3}}{2}, \frac{3}{2}\right), P_{4}=\left(-\frac{\sqrt{3}}{2}, \frac{3}{2}\right), P_{5}=(-\sqrt{3}, 0), P_{6}=\left(-\frac{\sqrt{3}}{2},-\frac{3}{2}\right), P_{7}=\left(\frac{\sqrt{3}}{2},-\frac{3}{2}\right)$ as depicted in Fig 4.I; the points $\mathrm{P}_{2}, \mathrm{P}_{3}, \mathrm{P}_{4}, \mathrm{P}_{5}, \mathrm{P}_{6}, \mathrm{P}_{7}$ are the vertices of the regular hexagon of length $\sqrt{3}$ centered at the origin. We prove that these 7 points pierce $\mathcal{D}$.

Let $D_{i} \in \mathcal{D}$ be a disk with center $c_{i}$ and radius $r_{i}$. Since $D_{1}$ is the smallest disk in $\mathcal{D}$, we have that $r_{i} \geqslant 1$. Since points $P_{2}, P_{3}, \ldots, P_{7}$ are the vertices of a regular hexagon, there must exist a $j \in\{2,3, \ldots, 7\}$ such that $\angle \mathrm{P}_{j} \mathrm{P}_{1} \mathrm{c}_{i} \leqslant \frac{\pi}{6}$. Let $\theta=\angle \mathrm{P}_{j} \mathrm{P}_{1} \mathrm{c}_{i}$. By the law of cosines,

$$
\left|c_{i} P_{j}\right|^{2}=\left|c_{i} P_{1}\right|^{2}+\left|P_{1} P_{j}\right|^{2}-2\left|c_{i} P_{1} \| P_{1} P_{j}\right| \cos (\theta)
$$

$\left|P_{1} P_{j}\right|=\sqrt{3}$ since these points all have distance $\sqrt{3}$ to the origin. $\left|c_{i} P_{1}\right| \leqslant r_{i}+1$ since $D_{i}$ and $D_{1}$ intersect. We have that $\cos (\theta) \geqslant$ 


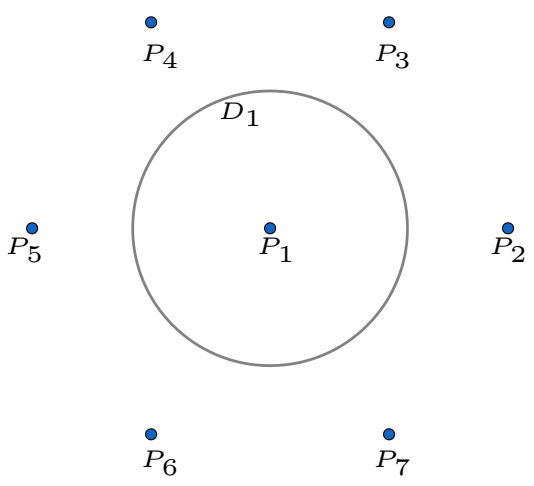

Figure 4.1: $\left\{\mathrm{P}_{1}, \mathrm{P}_{2}, \mathrm{P}_{3}, \mathrm{P}_{4}, \mathrm{P}_{5}, \mathrm{P}_{6}, \mathrm{P}_{7}\right\}$ pierce any disk with radius $\geqslant 1$ and intersects $D_{1}$.

$\cos \left(\frac{\pi}{6}\right)$ since $\theta \leqslant \frac{\pi}{6}$. Therefore, $-2\left|c_{i} P_{1}\left\|P_{1} P_{j}|\cos (\theta) \leqslant-2| c_{i} P_{1}\right\| P_{1} P_{j}\right| \cos \left(\frac{\pi}{6}\right)$. By replacing terms in the equation, we get

$$
\left|c_{i} P_{j}\right|^{2} \leqslant\left|c_{i} P_{1}\right|^{2}+(\sqrt{3})^{2}-2 \sqrt{3}\left|c_{i} P_{1}\right| \cos \left(\frac{\pi}{6}\right)
$$

By simplification, we get

$$
\left|c_{i} P_{j}\right|^{2} \leqslant\left|c_{i} P_{1}\right|^{2}+3-3\left|c_{i} P_{1}\right|
$$

By rearranging terms in the equation, we get

$$
\left|c_{i} P_{j}\right|^{2} \leqslant\left(\left|c_{i} P_{1}\right|-1\right)^{2}-\left|c_{i} P_{1}\right|+2
$$

When $\left|c_{i} P_{1}\right| \geqslant 2,\left(\left|c_{i} P_{1}\right|-1\right)^{2}-\left|c_{i} P_{1}\right|+2 \leqslant r_{i}^{2}+2-\left|c_{i} P_{1}\right| \leqslant r_{i}^{2}$. Therefore, $\left|c_{i} P_{j}\right| \leqslant r_{i}$ and $D_{i}$ contains $P_{j}$. If $\left|c_{i} P_{1}\right| \leqslant 1, c_{i}$ falls in $D_{1}$. Then $D_{i}$ is pierced by $P_{1}$ since $r_{i} \geqslant 1$.

Now let us consider the case when $1<\left|c_{i} P_{1}\right|<2$. Let $f(x)$ be the parabola $x^{2}-3 x+3$. The vertex of $f(x)$ is $\left(\frac{3}{2}, \frac{3}{4}\right)$. Therefore, when $1<x \leqslant \frac{3}{2}, \frac{3}{4} \leqslant f(x)<1$. Similarly, when $\frac{3}{2} \leqslant x<2, \frac{3}{4} \leqslant f(x)<1$. Combining these results together, we have that $f(x)<1$ when $1<$ $x<2$. Let $\left|c_{i} P_{1}\right|=x$, then we have that $\left|c_{i} P_{j}\right|^{2} \leqslant f(x)<1$. Therefore, $\left|c_{i} P_{j}\right|<1$ and $P_{j}$ pierces $D_{i}$ since $r_{i} \geqslant 1$.

\subsection{PIERCING WITH FIVE POINTS}

We now show how to pierce a set $\mathcal{D}$ of pairwise intersecting disks of arbitrary radius with 5 points. Let $\mathrm{D}_{1}$ be the smallest disk in $\mathcal{D}$. We shrink $D_{1}$ while keeping its center fixed at $c_{1}$ until $D_{1}$ becomes tangent to another disk, say $\mathrm{D}_{2}$. In the new setting, disks in $\mathcal{D}$ are still pairwise intersecting and any set of points that pierces the new set of disks also pierces the original set of disks. By scaling, rotation 


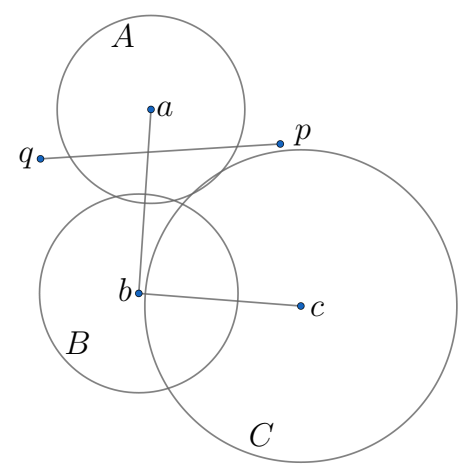

Figure 4.2: $\{A, B, C\}$ are three pairwise intersecting disks. $A$ intersects $B$ between $p$ and $q$. $C$ intersects $B$, but not between $p$ and $q$ since $p q$ and bc do not cross.

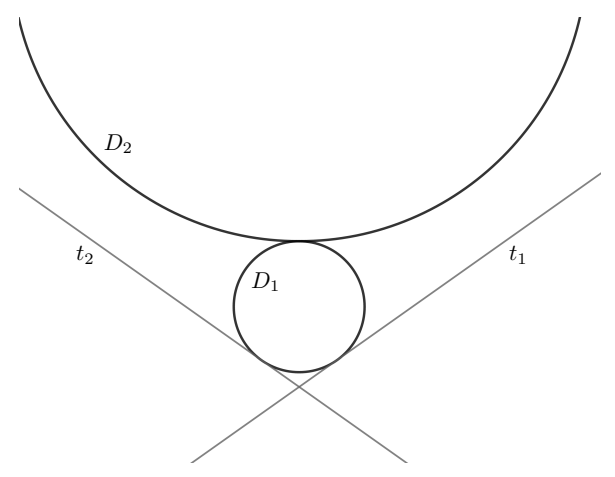

Figure 4.3: Configuration of lemma 4.3.

and translation, we assume $D_{1}$ is centered at the origin with radius 1 and $D_{2}$ is centered on the positive $y$-axis. Let $P_{1}, P_{2}, P_{3}, P_{4}, P_{5}$ be the points from the proof of Theorem 4.1 and let $P=\left\{P_{1}, P_{2}, P_{3}, P_{4}, P_{5}\right\}$.

We first define what it means for a disk to intersect another disk between two points in the plane; see Figure 4.2.

DEFINITION 4.2 (Between). Let A and B be two intersecting disks, and let $\mathrm{p}$ and $\mathrm{q}$ be two points in the plane. Let the center of $\mathrm{A}$ (resp. B) be a (resp. b). We say that A intersects B between $\mathrm{p}$ and $\mathrm{q}$ if the following two conditions hold:

- Line segment ab intersects line segment pq.

- Both $\mathrm{p}$ and $\mathrm{q}$ do not lie within A.

Before we present our algorithm for computing the piercing point set, we first present two geometric lemmas that will be proved in Section 4.2.1 and Section 4.2.2, respectively. See Figure 4.3 for the configuration outlined in the statement of lemma 4.3 and Figure 4.4 for the configuration of lemma $4 \cdot 4$. 


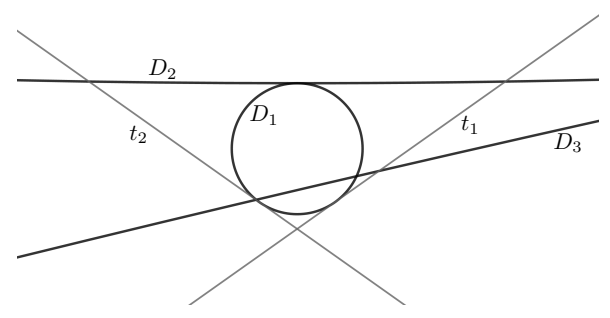

Figure 4.4: Configuration of lemma 4.4.

LEMMA 4.3. Let $\mathcal{D}$ be a set of pairwise intersecting disks of arbitrary radius such that its smallest disk $\mathrm{D}_{1}$ is tangent to a disk $\mathrm{D}_{2} \in \mathcal{D}$. If the radius of $\mathrm{D}_{1}$ is 1 and the radius of $\mathrm{D}_{2}$ is at most $5+2 \sqrt{6}$, then $\mathrm{P}$ pierces $\mathcal{D}$.

LEMMA 4.4. Let $\mathcal{D}$ be a set of pairwise intersecting disks of arbitrary radius such that its smallest disk $\mathrm{D}_{1}$ is tangent to a disk $\mathrm{D}_{2} \in \mathcal{D}$. If the radius of $\mathrm{D}_{1}$ is 1 , the radius of $\mathrm{D}_{2}$ is larger than $5+2 \sqrt{6}$ and there exist at least a disk in $\mathcal{D}$ that misses all the points in $\mathrm{P}$, then we can find in constant time a different set of 5 points that pierces $\mathcal{D}$.

These two lemmas are sufficient for proving the existence of 5 piercing points for pairwise intersecting arbitrary disks. The main contribution is that these points can be computed in linear time without the use of the LP-type machinery.

THEOREM 4.5. Given a set of pairwise intersecting arbitrary disks in the plane, in deterministic linear time, we can find 5 points that pierce the set.

Proof. Let $\mathcal{D}$ be the set of pairwise intersecting arbitrary disks in the plane. We find, in linear time, the smallest disk $D_{1} \in \mathcal{D}$. We compute the distance from $c_{1}$ to all disks in $\mathcal{D}$. We find the disk $D_{2} \in \mathcal{D}$ where the distance from $c_{1}$ to $D_{2}$ is the smallest. We shrink $r_{1}$, while keeping $D_{1}$ 's center fixed, by $\left|c_{1} c_{2}\right|-r_{2}$. After shrinking, $D_{1}$ becomes tangent to $D_{2}$ and the disks in $\mathcal{D}$ are still pairwise intersecting. With a proper scaling, rotation, and translation, assume $D_{1}$ has radius 1 and is centered at the origin and $\mathrm{D}_{2}$ is centered on the positive $y$-axis; these transformations can be performed in linear time.

If $r_{2} \leqslant 5+2 \sqrt{6}$, then by lemma $4 \cdot 3$, $P$ pierces $\mathcal{D}$. If $r_{2}>5+2 \sqrt{6}$, then we first check whether $P$ pierces $\mathcal{D}$. This step also takes linear time since for each disk $D_{i} \in \mathcal{D}$, we can check in constant time whether one of these 5 points is contained in $D_{i}$. If there exists at least one disk in $\mathcal{D}$ that is not pierced by any of these 5 points, we can then apply lemma 4.4 and compute a new candidate set of 5 points. We show in the proof of lemma 4.4 that these 5 points pierce $\mathcal{D}$ and the coordinate of these points can be computed in constant time.

Since we only shrank $\mathrm{D}_{1}$ in order to compute these piercing points, the 5 computed points pierce the origin set of disks. Each of these 


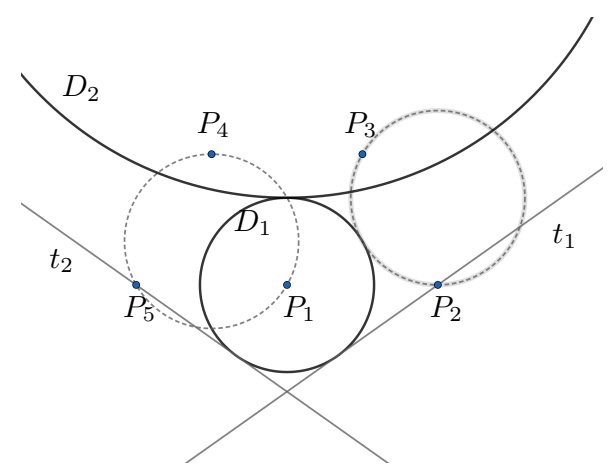

Figure 4.5: The first candidate set of 5 points.

steps takes either constant time or linear time, so the algorithm runs in linear time.

\subsubsection{Proof for Lemma 4.3}

In this section, we prove lemma 4.3. Recall that we assume the smallest disk $D_{1} \in \mathcal{D}$ is tangent to a second disk $D_{2} \in \mathcal{D}$. This can be ensured by shrinking the radius of $\mathrm{D}_{1}$ while keeping its center fixed. By a proper scaling, rotation, and translation, we assume $c_{1}$ is the origin, $c_{2}$ lies on the positive $y$-axis and $r_{1}=1$. $P$ is the set containing the following five points: $P_{1}=(0,0), P_{2}=(\sqrt{3}, 0), P_{3}=\left(\frac{\sqrt{3}}{2}, \frac{3}{2}\right)$, $P_{4}=\left(-\frac{\sqrt{3}}{2}, \frac{3}{2}\right), P_{5}=(-\sqrt{3}, 0)$; see Fig 4.5 .

Proof. Let $t_{1}$ be the line that is tangent to $D_{1}$ and passing through $P_{2}$. The equation of $t_{1}$ is $\frac{\sqrt{2}}{2} x-\frac{\sqrt{6}}{2}$. Let $t_{2}$ be the line that is tangent to $D_{1}$ and passing through $P_{5}$. The equation of $t_{2}$ is $-\frac{\sqrt{2}}{2} x-\frac{\sqrt{6}}{2} \cdot D_{2}$ is centered at the point $\left(0, r_{2}+1\right)$ since $r_{1}=1$. $D_{2}$ is tangent to both $t_{1}$ and $t_{2}$ when $r=5+2 \sqrt{6}$. Therefore, when $r_{2} \leqslant 5+2 \sqrt{6}, D_{2}$ lies completely above $t_{1}$ and $t_{2}$. We assume that our plane is a Cartesian plane, so the four quadrants are numbered counterclockwisely from 1 to 4 with the upper right corner being the first quadrant. Using an argument similar to the proof of Theorem 4.I, we can prove that any disk in $\mathcal{D}$ whose center falls in the first or the second quadrant is pierced by one of the points in $P$.

We now show that any disk in $\mathcal{D}$ whose center falls in the third or fourth quadrant is pierced by at least one of $\left\{\mathrm{P}_{1}, \mathrm{P}_{2}, \mathrm{P}_{5}\right\}$. If all disks are pierced by at least one of these points, then we are done. So we assume that there exists at least one disk, say $D_{3}$, that is not pierced by any of these three points. Since $D_{2}$ lies completely above $t_{1}$ and $t_{2}$, $D_{3}$ must intersect $D_{2}$ between $P_{1}$ and $P_{2}$ or between $P_{1}$ and $P_{5} . D_{3}$ 's radius is at least 1 since otherwise it contradicts the assumption that $D_{1}$ is the smallest disk in $\mathcal{D}$. Then $D_{3}$ do not cross the $y=1$ line. $D_{2}$ lies completely above the $y=1$ line, so $D_{3}$ does not intersect $D_{2}$ and 


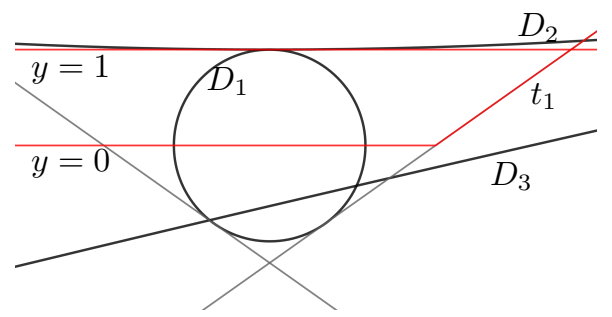

Figure 4.6: Boundaries that disks in $\mathcal{D}$ need to cross.

we have a contradiction. Therefore, any disk in $\mathcal{D}$ whose center falls in the third or fourth quadrant is pierced by one of $\left\{\mathrm{P}_{1}, \mathrm{P}_{2}, \mathrm{P}_{5}\right\}$.

\subsubsection{Proof for Lemma 4.4}

In this section, we will prove the lemma 4.4. We assume the same setting as in Section 4.2.1.

Proof. Recall the lines $t_{1}, t_{2}$, and the point set $P$ from the proof of lemma 4.3. Since $r_{2}>5+2 \sqrt{6}, D_{2}$ intersects both $t_{1}$ and $t_{2}$. We assumed that there exists at least one disk, say $D_{3} \in \mathcal{D}$ that is not pierced by $P . D_{3}$ intersects both $D_{1}$ and $D_{2}$. The center $c_{3}$ of $D_{3}$ cannot lie in the first or second quadrant since otherwise it must contain one point of $\mathrm{P}$ as shown in the proof of Theorem 4.1. Up to symmetry we may assume that the center $c_{3}$ lies in the fourth quadrant, and thus it intersects $D_{2}$ to the right side of the $y$-axis. This setting is depicted in Figure 4.6.

Since the interior of $D_{1}$ lies completely below the line $y=1$ and the interior of $D_{2}$ lies completely above this line, any disk in $\mathcal{D} \backslash\left\{D_{1}, D_{2}\right\}$ must cross this line in order to intersect both $D_{1}$ and $D_{2}$. Since $D_{3}$ misses $\mathrm{P}$, then $\mathrm{D}_{3}$ must lie completely below the polygonal line

$$
\ell: \begin{cases}y=0, & x \leqslant \sqrt{3} \\ t_{1}, & x>\sqrt{3}\end{cases}
$$

as shown in Figure 4.6. Any disk in $\mathcal{D}$ whose center falls above $\ell$ must cross $\ell$ in order to intersect with $\mathrm{D}_{3}$.

We are going to construct a point set $\mathrm{P}^{\prime}=\left\{\mathrm{P}_{6}, \mathrm{P}_{7}, \mathrm{P}_{8}, \mathrm{P}_{9}, \mathrm{P}_{10}\right\}$ that pierces $\mathcal{D}$. Set $P_{6}=(0,-3)$. In the rest of the proof we describe how to obtain $\mathrm{P}_{7}, \mathrm{P}_{8}, \mathrm{P}_{9}$, and $\mathrm{P}_{10}$; the coordinates of these points are given in Section 4.2.3 Let $C_{1}$ (resp. $C_{2}$ ) be the circle passing through $P_{6}$ that is tangent to disk $D_{1}$ and line $y=1$ in the left side (resp. right side) of the $y$-axis, as in Fig 4.7. Let $C_{3}$ be the circle that is centered above $y=1$ and that is tangent to the disk $D_{1}$, the line $t_{1}$ and to the x-axis. The disks $C_{1}$ and $C_{3}$ intersect at two points, where we pick 


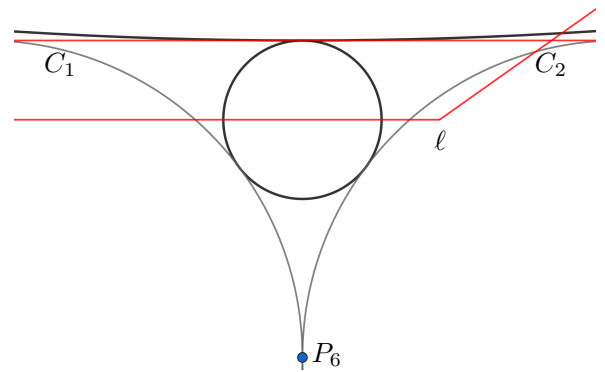

Figure 4.7: The location of $\mathrm{P}_{6}$.

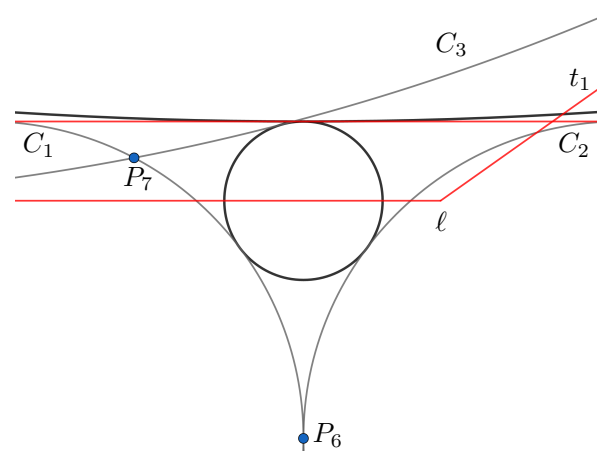

Figure 4.8: The location of $\mathrm{P}_{7}$.

the intersection point that is closer to the origin as the point $\mathrm{P}_{7}$; see Fig 4.8.

Now let $C_{4}$ be a circle of radius 1 that passes though $P_{7}$ and that is tangent to the $x$-axis, and let $C_{5}$ be a circle of radius 1 that passes through $P_{7}$ and that is tangent to the the line $y=1$. The point $P_{8}$ is the intersection point between $C_{4}$ and $C_{5}$ that is different from $P_{7}$. See Fig 4.9 for an illustration.

To obtain $P_{9}$, let $C_{6}$ be a circle of radius 1 that passes through $P_{8}$ and that is tangent to the line $y=1$. The intersection point of $C_{2}$ and $\mathrm{C}_{6}$ that falls in the first quadrant is $\mathrm{P}_{9}$, as depicted in Fig 4.10.

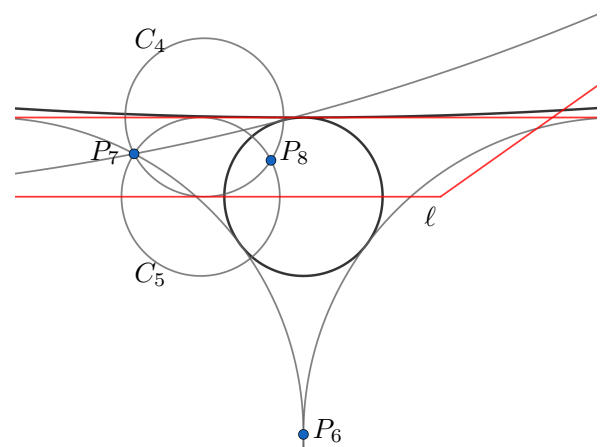

Figure 4.9: The location of $\mathrm{P}_{8}$. 


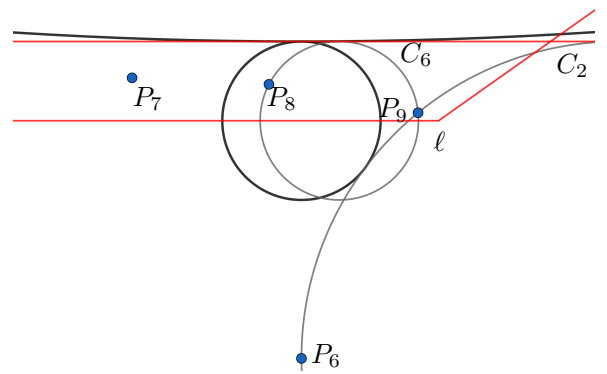

Figure 4.10: The location of $\mathrm{P}_{9}$.

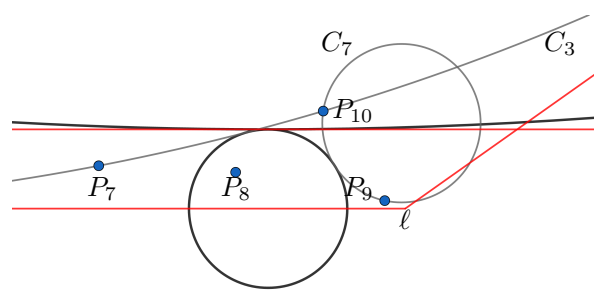

- $P_{6}$

Figure 4.11: The location of $\mathrm{P}_{10}$.

To obtain $\mathrm{P}_{10}$, we draw a circle $\mathrm{C}_{7}$ of radius 1 through $\mathrm{P}_{9}$ and tangent to $D_{1}$. The point $P_{10}$ is the intersection point of $C_{3}$ and $C_{7}$ that is closer to the origin, as in Fig 4.10.

Now that all five points in $\mathrm{P}^{\prime}$ have been introduced, we are going to show that these five points pierce all disks in $\mathcal{D}$. Although this is implicit from our construction of $\mathrm{P}^{\prime}$, we will describe this explicitly.

Consider the convex quadrilateral formed by $\mathrm{P}_{6}, \mathrm{P}_{7}, \mathrm{P}_{9}, \mathrm{P}_{10}$, as in Fig 4.12. These four points pierce any disk of $\mathcal{D}$ whose center lies outside the quadrilateral, because any such disk should intersect $D_{1}$.

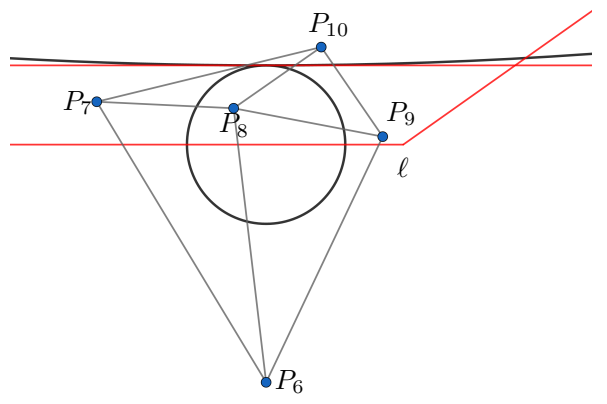

Figure 4.12: $P_{6}, P_{7}, P_{9}, P_{10}$ forms a quadrilateral around $D_{1}$. 
- $C_{3}$ is tangent to $\ell$ and $D_{1}$, and both $P_{7}$ and $P_{10}$ lie on $C_{3}$. If a disk $\mathrm{D}_{4}$ in $\mathcal{D}$ intersects $\mathrm{D}_{1}$ between $\mathrm{P}_{7}$ and $\mathrm{P}_{10}, \mathrm{D}_{4}$ cannot cross $\ell$. Since $\mathrm{D}_{3}$ lies completely below $\ell$, so $\mathrm{D}_{4}$ do not intersect $\mathrm{D}_{3}$ and it violates the pairwise intersecting property of $\mathcal{D}$.

- Both $P_{6}$ and $P_{7}$ lie on $C_{1}$, and $C_{1}$ is tangent to the $y=1$ line. If a disk $D_{4}$ intersects $D_{1}$ between $P_{6}$ and $P_{7}$, then $D_{4}$ do not intersect $\mathrm{D}_{2}$ and again contradicts our assumption that the disks in $\mathcal{D}$ are pairwise intersecting. Using a similar argument, we can also prove that there cannot exist a disk in $\mathcal{D}$ that intersects $D_{1}$ between $\mathrm{P}_{6}$ and $\mathrm{P}_{9}$.

- Any disk that intersects with $\mathrm{D}_{1}$ between $\mathrm{P}_{9}$ and $\mathrm{P}_{10}$ must contain one of these two points. Otherwise, its radius is smaller than 1 , contradicting the fact that $\mathrm{D}_{1}$ is the smallest disk.

Now we show how the disks of $\mathcal{D}$ centered inside the quadrilateral are pierced by points in $\mathrm{P}^{\prime}$. We divide the quadrilateral into four triangles, as in Fig 4.12.

- $P_{7}$ and $P_{8}$ both lie on $C_{5}$ and the radius of $C_{5}$ is 1 . Therefore, any disk whose center lies in $\triangle \mathrm{P}_{6} \mathrm{P}_{7} \mathrm{P}_{8}$ must contain one of $P_{7}$ or $P_{8}$ in order to intersect with $D_{2}$, otherwise its radius is smaller than 1 .

- Similarly, $\mathrm{P}_{7}$ and $\mathrm{P}_{8}$ both lie on $\mathrm{C}_{4}$ and the radius of $\mathrm{c}_{4}$ is also 1 . Therefore, any disk whose center lies in $\triangle \mathrm{P}_{7} \mathrm{P}_{8} \mathrm{P}_{10}$ must contain one of $P_{7}$ and $P_{8}$ in order to intersect with $D_{3}$.

- Any disk whose center lies in $\triangle \mathrm{P}_{8} \mathrm{P}_{9} \mathrm{P}_{10}$ must contain one of these three vertices because the diameter of this triangle is at most 2 .

- Any disk whose center falls in $\triangle \mathrm{P}_{6} \mathrm{P}_{8} \mathrm{P}_{9}$ must contain one of $\mathrm{P}_{8}$ and $P_{9}$ in order to intersect $D_{2}$, otherwise its radius is smaller than 1 since $C_{6}$ has radius 1 and both $P_{8}$ and $P_{9}$ lie on $C_{6}$.

Having $D_{1}, D_{2}, t_{1}$, and $t_{2}$, the point set $P^{\prime}$ can be found in constant time.

\subsubsection{Coordinates of Points in Lemma 4.4}

Recall that $D_{1}$ is the smallest disk in $\mathcal{D}$ with radius 1 , and it is centered at the origin. $D_{1}$ is tangent to a disk $D_{2} \in \mathcal{D}$, where $D_{2}$ centered on the positive $y$-axis. There exists a disk $D_{3} \in \mathcal{D}$ whose center falls in the fourth quadrant and misses the set of 5 points in $P$ as listed in lemma $4 \cdot 3$.

For each point $P_{i}$, let $x_{i}$ be its $x$-coordinate and $y_{i}$ be its $y$-coordinate, and for each circle $C_{i}$, let $\left(x_{i}^{\prime}, y_{i}^{\prime}\right)$ be its center and $r_{i}^{\prime}$ be its radius. 
$\mathrm{P}_{6}$ 's coordinate is $(0,-3)$.

$C_{1}$ can be represented by $(x+4)^{2}+(y+3)^{2}=16$.

$C_{2}$ can be represented by $(x-4)^{2}+(y+3)^{2}=16$.

$\mathrm{C}_{3}$ can be represented by $\left(x-x_{3}^{\prime}\right)^{2}+\left(y-y_{3}^{\prime}\right)^{2}=\left(r_{3}^{\prime}\right)^{2}$, where $x_{3}^{\prime}=$ $-\sqrt{1+2 r_{3}^{\prime}}, y_{3}^{\prime}=r_{3}^{\prime}, r_{3}^{\prime}=\frac{16-4 \sqrt{6}+\sqrt{(16-4 \sqrt{6})^{2}-16(\sqrt{6}-2)^{2}}}{2(\sqrt{6}-2)^{2}}$

$P_{7}$ 's coordinate is $\left(\frac{\left(-2 r_{3}^{\prime}-6\right) y_{7}+\left(x_{3}^{\prime}\right)^{2}-9}{2 x_{3}^{\prime}+8}, \frac{-b_{1}+\sqrt{b_{1}^{2}-4 a_{1} c_{1}}}{2 a_{1}}\right)$ where $a_{1}=$ $\left(-2 r_{3}^{\prime}-6\right)^{2}+\left(2 x_{3}^{\prime}+8\right)^{2}, b_{1}=2\left(-2 r_{3}^{\prime}-6\right)\left(\left(x_{3}^{\prime}\right)^{2}-9\right)+8\left(2 x_{3}^{\prime}+8\right)\left(-2 r_{3}^{\prime}-\right.$ 6) $+6\left(2 x_{3}^{\prime}+8\right)^{2}, c_{1}=\left(\left(x_{3}^{\prime}\right)^{2}-9\right)^{2}+8\left(2 x_{3}^{\prime}+8\right)\left(\left(x_{3}^{\prime}\right)^{2}-9\right)+9\left(2 x_{3}^{\prime}+\right.$ $8)^{2}$.

$C_{4}$ 's equation is $\left(x-\sqrt{2 y_{7}-y_{7}^{2}}-x_{7}\right)^{2}+(y-1)^{2}=1$.

$C_{5}$ 's equation is $\left(x-\sqrt{1-y_{7}^{2}}-x_{7}\right)^{2}+y^{2}=1$.

$P_{8}$ 's coordinate is $\left(\frac{2 y_{8}+q_{1}}{q_{2}}, \frac{-b_{2}-\sqrt{b_{2}^{2}-4 a_{2} c_{2}}}{2 a_{2}}\right)$ where $q_{1}=\left(x_{5}^{\prime}\right)^{2}-$ $\left(x_{4}^{\prime}\right)^{2}-1, q_{2}=2 x_{5}^{\prime}-2 x_{4}^{\prime}, a_{2}=4+q_{2}^{2}, b_{2}=4 q_{1}-4 q_{2} x_{5}^{\prime}, c_{2}=q_{1}^{2}+$ $\mathrm{q}_{2}^{2}\left(x_{5}^{\prime}\right)^{2}-2 \mathrm{q}_{1} \mathrm{q}_{2} x_{5}^{\prime}-\mathrm{q}_{2}^{2}$.

$C_{6}$ 's equation is $\left(x-\sqrt{1-y_{8}^{2}}-x_{8}\right)^{2}+y^{2}=1$.

$P_{9}$ 's coordinate is $\left(\frac{-b_{3}+\sqrt{b_{3}^{2}-4 a_{3} c_{3}}}{2 a_{3}}, \frac{q_{3} x_{9}+q_{4}}{6}\right)$ where $q_{3}=8-2 x_{6}^{\prime}, q_{4}=$ $\left(x_{6}^{\prime}\right)^{2}-10, a_{3}=36+q_{3}^{2}, b_{3}=2 q_{3} q_{4}+36 q_{3}-288, c_{3}=q_{4}^{2}+36 q_{4}+$ 324.

$C_{7}$ 's center is $\left(\sqrt{4-\left(y_{7}^{\prime}\right)^{2}}, \frac{-b_{4}+\sqrt{b_{4}^{2}-4 a_{4} c_{4}}}{2 a_{4}}\right)$ where $a_{4}=4 x_{9}^{2}+4 y_{9}^{2}, b_{4}=$ $-4 y_{9}\left(x_{9}^{2}+y_{9}^{2}+3\right), c_{4}=\left(x_{9}^{2}+y_{9}^{2}+3\right)^{2}-16 x_{9}^{2}$.

$P_{10}$ 's coordinate is $\left(x_{7}^{\prime}-\sqrt{1-\left(y_{10}-y_{7}^{\prime}\right)^{2}}, \frac{-b_{5}-\sqrt{b_{5}^{2}-4 a_{5} c_{5}}}{2 a_{5}}\right)$ where $q_{5}=\left(x_{7}^{\prime}\right)^{2}+\left(y_{7}^{\prime}\right)^{2}-\left(x_{3}^{\prime}\right)^{2}-\left(y_{3}^{\prime}\right)^{2}+\left(r_{3}^{\prime}\right)^{2}-1-\left(2 x_{7}^{\prime}-2 x_{3}^{\prime}\right) x_{7}^{\prime}, a_{5}=$ $\left(2 y_{3}^{\prime}-2 y_{7}^{\prime}\right)^{2}+\left(2 x_{7}^{\prime}-2 x_{3}^{\prime}\right)^{2}, b_{5}=2 q_{5}\left(2 y_{3}^{\prime}-2 y_{7}^{\prime}\right)-2 y_{7}^{\prime}\left(2 x_{7}^{\prime}-2 x_{3}^{\prime}\right)^{2}, c_{5}=$ $\mathrm{q}_{5}^{2}+\left(\left(y_{7}^{\prime}\right)^{2}-1\right)\left(2 x_{7}^{\prime}-2 x_{3}^{\prime}\right)^{2}$. 
We start the concluding chapter with a summary of contributions of this thesis, namely the simple algorithms that we presented in Chapter 3 and Chapter 4. Then we discuss the results and finally end this chapter by mentioning directions for future research.

\subsection{SUMMARY OF CONTRIBUTIONS}

In this thesis, we presented two simple deterministic linear-time algorithms for piercing pairwise intersecting unit disks. We also presented a simple linear-time algorithm for piercing pairwise intersecting arbitrary disks. In addition, we presented a lower case construction of 9 pairwise intersecting unit disks.

The first algorithm we proposed for piercing pairwise intersecting unit disks does not involve solving any LP-type problem. It simply shrinks one disk in the set until the disk becomes tangent to another disk. Using the $x$-coordinate of the rightmost disk in the set, we compute three points that cover the area in which the centers of all the disks in the set can fall. The second algorithm we proposed for piercing pairwise intersecting unit disks requires solving an LP-type problem, which is finding the smallest disk that intersects every disk in the set. Once the smallest disk is computed, we can immediately suggest three points whose coordinates are computed with respect to the center of the smallest disk that intersects the set of disks. Both algorithms run in deterministic linear time.

The algorithm we proposed for piercing pairwise intersecting arbitrary disks does not build the set of piercing points based on a non-Helly triple or the smallest disk intersecting every disk in the set. Our algorithm shrinks the smallest disk in the set until it becomes tangent to a second disk. Then we suggest two candidate sets of 5 points. The coordinates of these points can be computed in constant time given the positions of the disks in the set. We proved that the set of disks is always pierced by one of the two candidate sets. The algorithm runs in linear time as it involves geometric transformations on the set of disks.

We also presented a set of 9 pairwise intersecting unit disks that cannot be pierced by 2 points. This lower bound construction completes the proof that 3 points are sometimes necessary and always sufficient to pierce pairwise intersecting disks of the same radius. 


\subsection{DISCUSSION}

The deterministic linear-time algorithms we proposed for piercing pairwise intersecting unit disks use different approaches compared to the argument given by Hadwiger and Debrunner [13]. Their construction is based on a regular hexagon with sides of length $\frac{2}{\sqrt{3}}$ that covers the centers of all the disks. They construct the piercing set by choosing three points that lie on the diagonals of the hexagon. It is not known whether we can find such a hexagon in linear time. The algorithm we presented in Section 3.1, on the other hand, uses simple geometric transformation and finds the piercing points using only the information of the disks in the set. Even though the other algorithm computes the piercing points based on the smallest disk intersecting every disk in the set, finding such disk can be formulated as an LP-type problem. Therefore, it can be solved in deterministic linear time [6].

Our deterministic linear-time algorithm for piercing pairwise intersecting arbitrary disks computes the coordinates of the piercing points without solving any LP-type problem. Even though the algorithm by Har-Peled et al. [15] runs in linear time, their algorithm depends on the smallest destroyer of the set. Finding the smallest destroyer is an LP-type problem. It can be solved in deterministic linear time since a constant time violation test is available for the problem and its range space has bounded VC-dimension. However, it requires effort to turn the algorithm for solving LP-type problems into an actual computer program. Our algorithm minimizes the effort required to turn itself into an actual computer program.

\section{$5 \cdot 3$ FUTURE WORK}

In this thesis, we have successfully shown how the piercing problem could be solved without involving any LP-type machinery. Many existing results about the piercing problem closely relate to LP-type problems. Therefore, there are still more works to do.

Carmi et al. [3] constructed a linear-time algorithm for piercing pairwise intersecting arbitrary disks with 4 points by using an LPtype problem as subroutine. Can we design an algorithm that finds a set of 4 points that pierces any set of disks without involving any LP-type problem? As can be seen from the algorithm we designed in Section 4.2.2, the polygonal line $\ell$ is essential when constructing the second set of 5 points. Since the first set of points is not sufficient, there must exist a disk that lies entirely below the polygonal line $\ell$. Therefore, one should be able to design an algorithm that suggests multiple candidate sets of 4 points. When one of these sets is not sufficient, then it implies a new polygonal line that needs to be crossed by the disks in 
the set. The new polygonal line can then be used for constructing the next candidate point set.

Another possible area for future research is determining the optimal number of points required to pierce the given set of disks. It is known that 3 (resp. 4) points are sometimes necessary and always sufficient for piercing pairwise intersecting unit disks (resp. arbitrary disks) $[13,8]$. However, fewer points are adequate for many sets. Therefore, can we design an efficient algorithm that can determine the optimal number of points required for piercing a given set of disks?

For the lower bound, Danzer [8] constructed a set of 10 disks that cannot be pierced by 3 points. We have showed in Section 2.4 that every set of 8 pairwise intersecting disks can be pierced by 3 points. So, the remaining question is: can every set of 9 pairwise intersecting disks of arbitrary radius be pierced by 3 points? In Section 3.3, we constructed a set of 9 pairwise intersecting unit disks that cannot be pierced by 2 points. Removal of any disk in the set will break this property. Can every set of 8 pairwise intersecting unit disks be pierced by 2 points? The answer to this question will prove or disprove that our lower bound construction is of minimum cardinality. 
BIBLIOGRAPHY

[1] N. Alon and D. J. Kleitman. Piercing convex sets and the Hadwiger-Debrunner (p, q)-problem. Advances in Mathematics, 96(1):103-112, 1992.

[2] P. Bose, P. Carmi, and T. Shermer. Piercing pairwise intersecting geodesic disks. Submitted to CGTA - Special Issue in Memoriam, Godfried Toussaint, 2020.

[3] P. Carmi, M. J. Katz, and P. Morin. Stabbing pairwise intersecting disks by four points, 2018. arXiv:1812.06907.

[4] T. Chan. An optimal randomized algorithm for maximum Tukey depth. pages 423-429, 2004. Copyright: Copyright 2008 Elsevier B.V., All rights reserved.; Proceedings of the Fifteenth Annual ACM-SIAM Symposium on Discrete Algorithms ; Conference date: 11-01-2004 Through 13-01-2004.

[5] B. Chazelle. The Discrepancy Method: Randomness and Complexity. Cambridge University Press, 2000.

[6] B. Chazelle and J. Matoušek. On linear-time deterministic algorithms for optimization problems in fixed dimension. Journal of Algorithms, 21(3):579 - 597, 1996.

[7] K. L. Clarkson, D. Eppstein, G. L. Miller, C. Sturtivant, and S. Teng. Approximating center points with iterated Radon points. In Proceedings of the Ninth Annual Symposium on Computational Geometry, SCG '93, pages 91-98, New York, NY, USA, 1993. Association for Computing Machinery.

[8] L. Danzer. Zur Lösung des Gallaischen Problems über Kreisscheiben in der Euklidischen Ebene. Studia Scientiarum Mathematicarum Hungarica, 21(1-2):111-134, 1986.

[9] L. Danzer, B. Grünbaum, and V. Klee. Helly's Theorem and Its Relatives. Proceedings of symposia in pure mathematics: Convexity. American Mathematical Society, 1963.

[10] P. Erdös and G. Szekeres. A combinatorial problem in geometry. Compositio Mathematica, 2:463-470, 1935.

[11] J. F. Grcar. How ordinary elimination became Gaussian elimination. Historia Mathematica, 38(2):163-218, 2011.

[12] B. Grünbaum. On intersections of similar sets. Portugal. Math., 18:155-164, 1959 .

[13] H. Hadwiger and H. Debrunner. Ausgewählte Einzelprobleme der kombinatorischen Geometrie in der Ebene. Enseignement Math. (2), 1:56-89, 1955. 
[14] S. Har-peled. Geometric Approximation Algorithms. American Mathematical Society, USA, 2011.

[15] S. Har-Peled, H. Kaplan, W. Mulzer, L. Roditty, P. Seiferth, M. Sharir, and M. Willert. Stabbing pairwise intersecting disks by five points. arXiv:1801.03158, 2018. Also in EuroCG'18.

[16] E. Helly. Über Mengen konvexer Körper mit gemeinschaftlichen Punkten. Jahresbericht der Deutschen Mathematiker-Vereinigung, 32:175-176, 1923 .

[17] D. König. Über konvexe körper. Mathematische Zeitschrift, 14(1):208-210, 1922.

[18] M. Löffler and M. van Kreveld. Largest bounding box, smallest diameter, and related problems on imprecise points. Computational Geometry, 43(4):419-433, 2010. Special Issue: 1oth Workshop on Algorithms and Data Structures (WADS 2007).

[19] J. Matoušek, M. Sharir, and E. Welzl. A subexponential bound for linear programming. In Proceedings of the Eighth Annual Symposium on Computational Geometry, SCG '92, pages 1-8, New York, NY, USA, 1992. Association for Computing Machinery.

[20] J. Radon. Mengen konvexer Körper, die einen gemeinsamen Punkt enthalten. Mathematische Annalen, 83(1):113-115, 1921.

[21] R. Seidel. Small-dimensional linear programming and convex hulls made easy. Discrete Comput. Geom., 6(5):423-434, Aug. 1991.

[22] M. Sharir and E. Welzl. A combinatorial bound for linear programming and related problems. In STACS, 1992.

[23] L. Stachó. A solution of Gallaiś problem on pinning down circles. Mat. Lapok, 32(1-3):19-47, 1981/84. 


\section{COLOPHON}

This document was typeset using the typographical look-and-feel classicthesis developed by André Miede. The style was inspired by Robert Bringhurst's seminal book on typography "The Elements of Typographic Style". classicthesis is available for both $\mathrm{LT}_{\mathrm{E}} \mathrm{X}$ and $\mathrm{LYX}$ :

http:/ / code.google.com/p/classicthesis/

Happy users of classicthesis usually send a real postcard to the author, a collection of postcards received so far is featured here:

$$
\text { http:/ / postcards.miede.de/ }
$$

Final Version as of May 18, 2021 (classicthesis version 4.1). 